\title{
Unexpected cell type-dependent effects of autophagy on polyglutamine aggregation revealed by natural genetic variation in C. elegans
}

\author{
J. Alexander-Floyd ${ }^{1,2}$, S. Haroon ${ }^{3}$, M. Ying ${ }^{1}$, A. A. Entezari ${ }^{1,4}$, C. Jaeger ${ }^{1,5}$, M. Vermulst ${ }^{3,6}$ and T. Gidalevitz ${ }^{1 *}$ (1)
}

\begin{abstract}
Background: Monogenic protein aggregation diseases, in addition to cell selectivity, exhibit clinical variation in the age of onset and progression, driven in part by inter-individual genetic variation. While natural genetic variants may pinpoint plastic networks amenable to intervention, the mechanisms by which they impact individual susceptibility to proteotoxicity are still largely unknown.
\end{abstract}

Results: We have previously shown that natural variation modifies polyglutamine (polyQ) aggregation phenotypes in C. elegans muscle cells. Here, we find that a genomic locus from C. elegans wild isolate DR1350 causes two genetically separable aggregation phenotypes, without changing the basal activity of muscle proteostasis pathways known to affect polyQ aggregation. We find that the increased aggregation phenotype was due to regulatory variants in the gene encoding a conserved autophagy protein ATG-5. The atg-5 gene itself conferred dosagedependent enhancement of aggregation, with the DR1350-derived allele behaving as hypermorph. Surprisingly, increased aggregation in animals carrying the modifier locus was accompanied by enhanced autophagy activation in response to activating treatment. Because autophagy is expected to clear, not increase, protein aggregates, we activated autophagy in three different polyQ models and found a striking tissue-dependent effect: activation of autophagy decreased polyQ aggregation in neurons and intestine, but increased it in the muscle cells.

Conclusions: Our data show that cryptic natural variants in genes encoding proteostasis components, although not causing detectable phenotypes in wild-type individuals, can have profound effects on aggregation-prone proteins. Clinical applications of autophagy activators for aggregation diseases may need to consider the unexpected divergent effects of autophagy in different cell types.

Keywords: Natural genetic variation, Regulatory variation, Cryptic variation, Protein aggregation, Proteostasis, Polyglutamine, Autophagy

\section{Background}

Protein misfolding and aggregation underlie many human diseases and contribute to tissue decline during aging $[1,2]$. In familial cases, the disease-causing mutations are often directly responsible for misfolding and aggregation of the mutant protein $[3,4]$. For example, expansions of the CAG repeats in several different diseases lead to expanded polyglutamine (polyQ) tracts in

\footnotetext{
* Correspondence: tg443@drexel.edu

${ }^{1}$ Biology Department, Drexel University, Philadelphia, PA 19104, USA Full list of author information is available at the end of the article
}

affected proteins, which in turn result in their increased aggregation propensity [5-7]. Such mutations exhibit "toxic gain-of-function" behavior and thus a dominant, monogenic inheritance pattern. The mechanisms explaining the gain-of-function toxicity are still incompletely understood. Two aspects of protein aggregation diseases may contribute to this difficulty. First, the behavior of mutant proteins appears to depend on the cellular environment: although they are often expressed broadly or even ubiquitously, only select subsets of cells are affected in each disease $[8,9]$. Second, these diseases

(c) The Author(s). 2020 Open Access This article is distributed under the terms of the Creative Commons Attribution 4.0 International License (http://creativecommons.org/licenses/by/4.0/), which permits unrestricted use, distribution, and reproduction in any medium, provided you give appropriate credit to the original author(s) and the source, provide a link to the Creative Commons license, and indicate if changes were made. The Creative Commons Public Domain Dedication waiver (http://creativecommons.org/publicdomain/zero/1.0/) applies to the data made available in this article, unless otherwise stated. 
show variation in the age of onset, severity, or clinical phenotypes [10]. The variation is thought to result, in addition to stochastic and environmental factors, from variants present in individual's genetic background that act as modifiers [11-13]. These genetic modifiers can affect proteins and regulatory pathways that either interact with the disease-causing mutant proteins, or are themselves impacted in disease [14]. Therefore, identifying natural modifier variants and their mechanisms can expand our understanding of cellular pathways involved in disease. Natural variants may also indicate pathways that differ from those found by the traditional approaches such as association studies, mutagenesis, or RNAi screens. Importantly, because these modifiers are a part of natural genetic variation and are present in phenotypically normal individuals, they may pinpoint therapeutic routes that are less likely to cause detrimental side effects.

The most informative way to map genetic modifiers of disease is directly in human patients [13]. A number of studies found that genetic variants other than those controlling the CAG repeat size of the polyQ-expanded huntingtin $(\mathrm{Htt})$ are capable of modifying the pathogenesis of Huntington's disease (HD) [12, 15-18]. Two recent large studies have identified four loci on chromosomes 3,8 , and 15 in HD subjects of European ancestry, and a locus on chromosome 7 in a Venezuelan HD cluster [19-21]. The modifier locus in Venezuelan HD may act by a novel mechanism-regulating the bone morphogenetic protein signaling, while pathway analysis in European HD implicated DNA repair pathways, which are thought to act by changing the size of the CAG repeat itself. The difficulties in using human patients in search for modifiers across aggregation diseases include the size and complexity of the human genome, the often small size of affected populations, and the possibility of complex interactions among multiple modifiers $[10,13,22]$. Human studies may also have limited ability to identify modifiers that are rare, or segregate in families rather than in entire affected populations. Model organisms offer a genetically tractable alternative due to the evolutionary conservation of the main cellular pathways. Expression of disease-related proteins in these organisms recapitulate many characteristics of human diseases that are related to the basic biology of protein misfolding and aggregation [23]. For example, $C$. elegans and Drosophila models expressing polyQexpanded $\mathrm{Htt}$ or ataxin-3, or isolated polyglutamine repeats, exhibit similar toxic gain-of-function behavior and the age- and polyQ-length-dependent aggregation and toxicity as those seen in patients and in mammalian models [24-34]. Many candidate modifying pathways identified in model organisms proved to be conserved, including insulin signaling, the heat-shock response, or regulators of proteostasis [35]. Importantly, as in human disease, polyQ expansions in C. elegans also exhibit dependence on both the cellular environment [30, 36, 37] and the genetic background [38], despite their dominant gain-of-function behavior. We have previously shown that genetic variants coding for marginally stable proteins, although innocuous under normal conditions, can dramatically change both the aggregation and the associated toxicity of the aggregation-prone proteins, suggesting that genetic variation may directly impinge on cellular proteostasis [37, 39]. Indeed, introduction of natural variation into the genetic background of polyQ-expressing animals independently modified several different aspects of polyQ behavior, including the onset and extent of aggregation, the susceptibility of different types of muscle cells to aggregation, and the resulting loss of motility and shortened lifespan [38]. The polyQ aggregation in these genetically variable animals showed transgressive segregation, indicating that multiple additive or interacting alleles in parental backgrounds were acting as modifiers [38]. A recent study has shown that natural variation also modulates the phenotypes caused by expression of $\alpha$-synuclein transgene in the body-wall muscle cells of $C$. elegans [40]. Thus, natural genetic variation within C. elegans wild strains can be used to investigate the mechanisms and pathways controlling the toxic effects of protein misfolding and aggregation.

Here, we dissected the genetic variation causing increased aggregation of the muscle-expressed 40-residue polyQ expansion (Q40::YFP, or Q40) in the background of a Californian wild strain of C. elegans, DR1350 [38]. We identified a large modifier locus on chromosome I as being causal for two phenotypes: altered susceptibility of the head muscle cells to aggregation and increased overall aggregation. These phenotypes were genetically separable, and we identified regulatory variants in a gene encoding a conserved autophagy protein ATG- 5 as being responsible for the latter phenotype. The atg-5 gene conferred a dosage-dependent enhancement of polyQ aggregation, with DR1350-derived atg-5 allele behaving as a hypermorph. Surprisingly, animals bearing the variant $\operatorname{atg}-5$ allele showed enhanced response to an autophagy-activating drug. Because autophagy is expected to clear polyQ aggregates, we tested the effect of directly activating autophagy on the polyQ aggregation in our model, and found a striking tissue dependence for the effect of autophagy on polyQ aggregation. Our data show that cryptic genetic variants in genes encoding proteostasis components can have profound effects on the behavior of aggregation-prone proteins, and suggest that activation of autophagy may have divergent effects on the clearance of such proteins in different cell types.

\section{Results}

DR1350-derived variants increase polyglutamine aggregation

We previously found that introgression of an integrated polyglutamine-encoding transgene (Q40) from the 
laboratory Bristol/N2 background (Q40Bristol) into the wild California isolate DR1350 resulted in strongly accelerated polyglutamine aggregation in the body-wall muscle cells, and a characteristic switch in the relative susceptibility of the normally resistant head muscle cells to polyQ aggregation [38]. These two phenotypes were also present in 5 out of 21 recombinant inbred lines (RILs) derived from a cross between Q40Bristol and Q40DR1350 strains [38]. The DR1350 parent belongs to the isotype defined by the California-derived strain CB4853 (the Caenorhabditis elegans Natural Diversity Resource [41]). Both strains have been used in some of the earliest studies on the effects of natural variation on phenotypic traits $[42,43]$, and DR1350 was also used to map quantitative trait loci (QTL) that control phenotypic responses to environmental stress [44]. Interestingly, genetic variation between DR1350 (or CB4853) and Bristol/N2 strains is unequally distributed across the chromosomes in C. elegans [41, 44, 45].
To isolate the genetic variation that contributed to increased aggregation, we chose one (RIL2) that exhibited more than twofold increase in the number of aggregates relative to the Q40Bristol parent at the late fourth larval stage (L4) (Fig. 1a). We backcrossed RIL2 animals to the Q40Bristol parental strain 23 times, selecting for the F2 progeny that inherited RIL2-like phenotypes after each round of backcrossing (Fig. 1b). This approach ensured that the DR1350-derived variants that contributed to the polyQ phenotypes were retained in the resulting $23 \times$ backcrossed strain, while the majority of its background was derived from the Q40Bristol parental strain. The backcrossed strain is referred to as $d r x I R 1 ; \mathrm{Q} 40$ (Fig. 1b). Since the increased susceptibility of the head muscles is an easy to detect qualitative phenotype that behaved in our RIL panel as a recessive trait [38], we used this phenotype during F2 progeny selection. Interestingly,

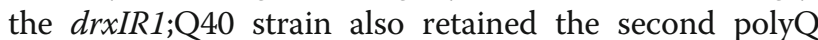
phenotype-increased overall aggregation (Fig. 1a, c),

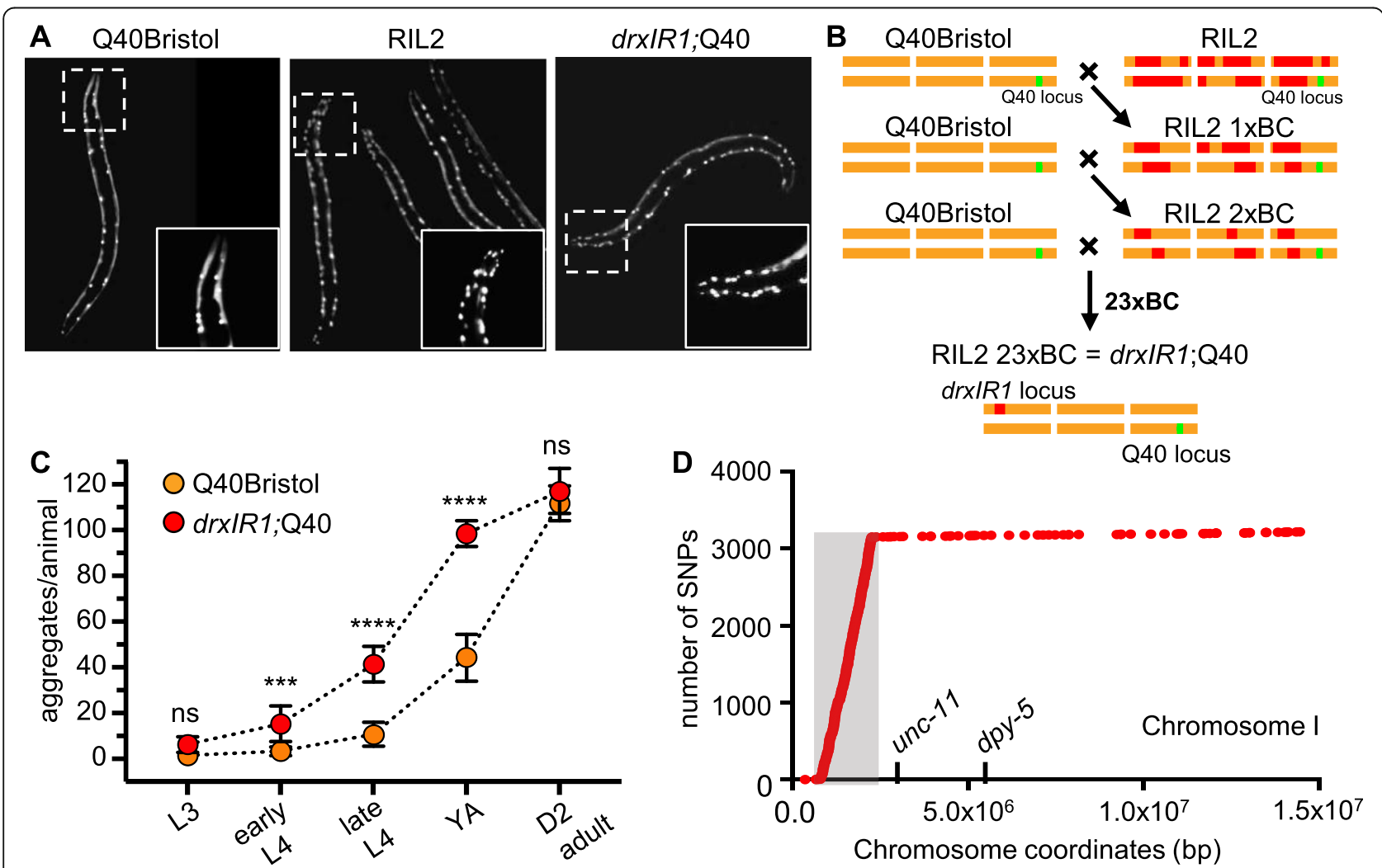

Fig. 1. $d r x I R 1$ locus causes increased polyQ40 aggregation. a Late-L4 RIL2 and drXIR1;Q40 animals have increased aggregation compared to Q40Bristol animals. Insets show polyQ40 aggregation in the head muscles. $\mathbf{b}$ The scheme for generation of the $d r x / R 7 ; \mathrm{Q} 40$ strain through rounds of serial backcrossing/selection. RIL2 strain was backcrossed (BC) into the Q40Bristol strain 23 times. DR1350-derived variants (red) that are retained through the crossing-selection scheme likely contribute to the RIL2 polyQ phenotype. c The drXIR1;Q40 animals exhibit a faster accumulation of polyQ aggregates compared to Q40Bristol at all development stages, until both strains reach maximum at day 2 of adulthood. L3, L4, YA, and D2 adult indicate third and fourth larval stage, young adult, and day 2 adult stage, respectively. Data are mean \pm SD, 10 to 20 animals per data point. Data were analyzed by ANOVA followed by Bonferroni's multiple comparisons test, ${ }^{* * *} P<0.0001,{ }^{* * *} P=0.0004$. Orange: Q40Bristol background, red: drxIR1;Q40. Same color scheme is used in all figures. d Distribution of DR1350-derived SNPs and any de novo mutations on chromosome I that distinguish drxIR1;Q40 from Q40Bristol and Hawaiian strains. Gray-shaded area to the left of unc-11 shows a locus with over 3000 unique SNPs in drXIR1;Q40 strain 
suggesting that the two phenotypes result from either linked or same natural variant(s). Age-matched $d r x I R 1$; Q40 animals had a higher number of polyQ40 aggregates than Q40Bristol until day 2 of adulthood, when polyQ40 aggregation reached maximum in both strains (Fig. 1c). drxIR1;Q40 animals also exhibited somewhat decreased motility at day 1 of adulthood (Additional file: Fig. S1A); however, we do not relate these observations to aggregation since we have previously showed that natural variation can uncouple aggregation from its associated toxic effects [38]. Thus, natural variants present in the wild isolate DR1350 can modify polyglutamine aggregation when introgressed into the Bristol genetic background.

\section{Polyglutamine aggregation-modifying variants reside in a} large interval inherited from the DR1350 parent

In order to identify the causative variant(s) in the backcrossed $d r x I R 1$; 40 strain, we first used mapping strains with visible mutations on each chromosome, and found that increased aggregation segregated with the left arm of chromosome I. This location was confirmed (described further below) using a free duplication sDP2 [46], which covers the left arm of chromosome I through dpy-5 (Additional file: Table S1). To precisely map the variant(s), we performed genome sequencing of both the $d r x I R 1 ; \mathrm{Q} 40$ and Q40Bristol strains and identified SNPs present only in the former, using Galaxy CloudMap pipeline described in [47]. We found that the left arm of chromosome I in the backcrossed $d r x I R 1 ; \mathrm{Q} 40$ strain contained an 1.43-Mb interval (ChrI:832,674-2,262,484), with over 4000 SNPs. Because our previous data showed that introgression of the Q40 transgene into the commonly used CB4856 (Hawaiian) strain did not result in the same aggregation phenotypes as in the DR1350 background [38], we used the list of known Hawaiian SNPs within the CloudMap pipeline [47] and subtracted them from the remaining $d r x I R 1 ; \mathrm{Q} 40$ SNPs. The genome of the Hawaiian strain is known to be highly divergent from the Bristol/N2 genome [45, 48]. After subtraction, the interval still contained over 3000 SNPs (Fig. 1d). We tested whether this interval was also present in the remaining four high-aggregation RILs from the original study, by following several SNPs within the interval (Additional file: Fig. S1). We found that three of the RILs indeed inherited the entire interval, while the interval in the fourth one (RIL15) was shorter on the right side, extending through SNP 6 at ChrI:1,850, 249 (WBVar00017051), but not through SNP 6b at ChrI:1, 972,719 (WBVar00017376) (Additional file: Fig. S1). Thus, 4 independent RILs with high polyQ aggregation phenotypes, and the 23 times back-crossed $d r x I R 1 ; \mathrm{Q} 40$ strain derived from another RIL (RIL2), all contained the parental interval ChrI:832,674-1,972,719 from the high- aggregation DR1350;Q40 strain. To confirm, we used a mutation in egl-30 gene located within this interval (Additional file: Fig. S1). Consistent with a close genetic linkage, we were unable to find any F2 progeny from 10 F1 heterozygotes from a cross between $d r x I R 1 ; \mathrm{Q} 40$ and egl-30(n686) animals that showed both the RIL2-like polyQ head aggregation phenotype and the egl phenotype ( $>1000$ F2s). Furthermore, in subsequent genetic crosses


a complete correlation between F2 progeny inheriting 2 copies of this interval, as detected by following SNP 5 (WBVar00016276) (see the "Methods" section), and the appearance of the 2 polyQ phenotypes ( $>100$ animals). Together, these data indicate that ChrI:832,674-1,972,719 interval is responsible for increased polyQ aggregation phenotypes.

The remaining part of chromosome I contained 68 additional SNPs relative to the Q40Bristol parental strain, and all the other chromosomes accumulated less than 200 unique SNPs each (Additional file: Fig. S2), consistent with previous reports [49]. The large size of the modifier interval was unexpected after 23 backcrosses, suggesting that it may contain structural variants preventing recombination over this region. Alternatively, this locus could contain more than one SNP responsible for the phenotypes, perhaps distributed over the interval. Of note, the known chromosome I zeel-1/peel-1 incompatibility locus [50] was not responsible for the retention of the modifier interval through the backcrosses, as it lays outside the mapped interval (Additional file: Fig. S1B), and does not contain DR1350-derived SNPs in the $d r x I R 1 ; \mathrm{Q} 40$ strain.

\section{Known regulators of proteostasis are not responsible for increased polyQ aggregation in drxIR1 animals}

Because the identified modifier locus contained a large number of SNPs, we thought to narrow down the candidate pathway(s) in which the modifier gene(s) acted. We first asked whether the variants in the $d r x I R 1$ locus were increasing polyglutamine aggregation by affecting either the protein homeostasis of the muscle cells, or the Q40:: YFP protein itself. We have previously tested and excluded the trivial explanation that the increased aggregation in our five RILs was due to the increased expression of the Q40::YFP protein [38]. Nonetheless, we considered a possibility that $d r x I R 1$ locus could cause increased activity of the unc-54 promoter that was used to drive the polyglutamine transgene. To test this, we introduced an integrated unc-54p::GFP::UNC-54 transgene [51] into the drxIR1 background, in the absence of polyQ, and examined its expression. We found no differences in the fluorescence levels, suggesting normal unc-54 promoter activity (Fig. 2a). Since assembly of myofilaments is sensitive to both the levels of UNC-54 myosin heavy chain 


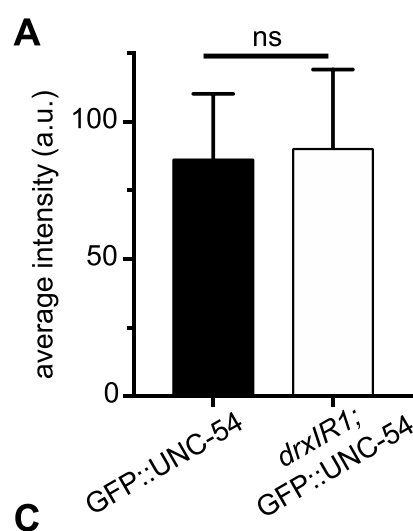

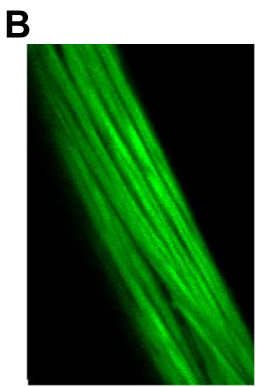

GFP::UNC-54

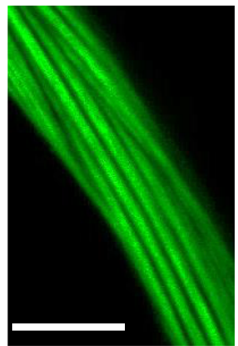

drxIR1;

GFP::UNC-54

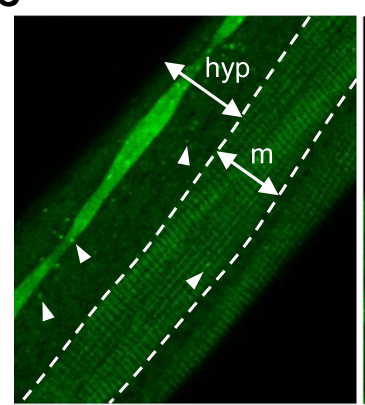

GFP::LGG-1
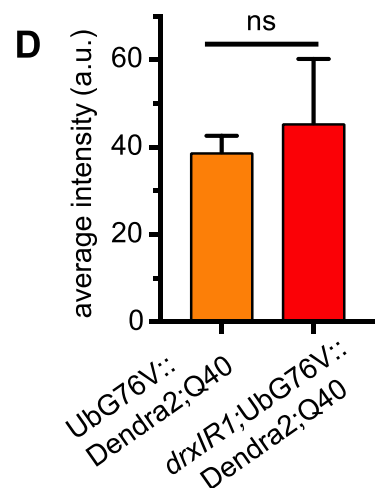

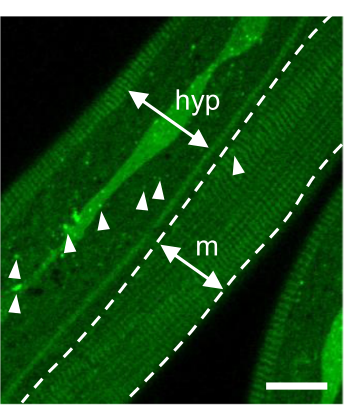

$d r \times I R 1 ; G F P:: L G G-1$

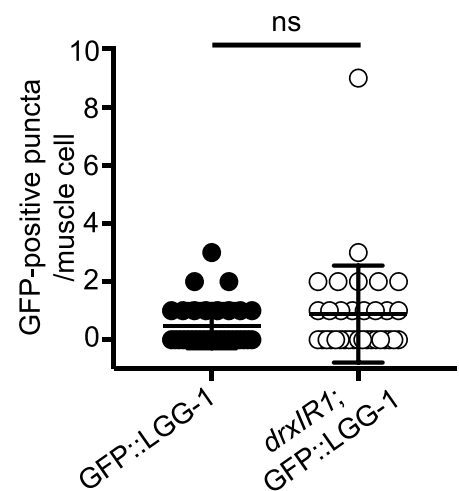

$\mathbf{E}$

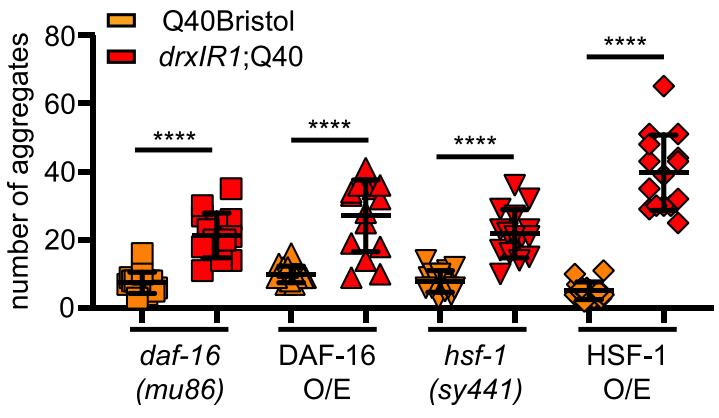

Fig. 2. Basal protein homeostasis of muscle cells is unaffected in animals carrying the drxIR1 interval. a Expression of GFP::UNC-54 fusion protein from unc-54 promoter is similar between the Bristol and drxIR 1 L4 animals. Data are mean \pm SD of GFP fluorescence intensity, 16-20 muscle cells per genotype, unpaired $t$ test, two-tailed. b Myofilament assembly is normal in drxIR1 animals. Confocal images of muscle cells. Scale bar, $10 \mu m$. c Muscle cells have very few GFP::LGG-1-positive puncta (arrowheads) in both Bristol and drxIR1 L4 animals. One muscle quadrant is shown between punctate lines. m, muscle; hyp, hypodermis. An increased number of GFP.:LGG-1-positive puncta is seen in the hypodermis of drxIR1. Scale bar is $10 \mu \mathrm{m}$. Right panel, quantification of GFP::LGG-1 puncta in the muscle cells. Data are mean \pm SD, 30 to 40 cells (8 to 10 animals) per genotype, unpaired $t$ test, two-tailed; each symbol represents individual cell. $\mathbf{d}$ No difference in the average intensity of the proteasome reporter fluorescence in Q40Bristol and drxIR1;Q40 animals. Data are mean \pm SD, 4-5 animals, unpaired $t$ test, two-tailed. e The increased aggregation phenotype in animals carrying the drxIR1 interval does not depend on DAF-16 or HSF-1. Each symbol represents an individual animal, 15 mid-L4 animals per genotype. O/E, overexpression. Means \pm SD are overlaid. Data were analyzed by ANOVA followed by Bonferroni's multiple comparisons test, ${ }^{* * * *} P<0.0001$

protein and the activity of molecular chaperones, it provides an additional measure of the GFP::UNC-54 protein levels and of the folding environment [52-54]. We found normal striated pattern of GFP::UNC-54 protein in both Bristol and drxIR1 genetic backgrounds (Fig. 2b).

Another reason for increased aggregation could be decreased protein turnover. To address this, we asked whether basal autophagy or proteasome activity was reduced in the muscle cells of $d r x I R 1$ animals. Using a well-characterized autophagy reporter ubiquitously expressing GFP::LGG-1 [55], GFP::LGG-1 puncta were counted in muscle cells of wild-type and $d r x I R 1$ animals, in the absence of Q40::YFP protein to avoid spectral overlap. Consistent with previously published results, the 
number of GFP-positive puncta in muscle cells of L4 animals with the Bristol background was low [56, 57], and we detected no difference in basal autophagy in the muscle cells of $d r x I R 1$ animals (Fig. 2c), although the increased number of puncta was noted in their lateral hypodermis. To test whether decreased proteasomal activity could be responsible for increased aggregation seen in the $d r x I R 1 ; \mathrm{Q} 40$ animals, we introduced a muscle-specific UbG76V::Dendra2 reporter [58] into Q40Bristol and drxIR1;Q40 animals, and measured its fluorescence. We detected no increase in Dendra2 fluorescence in $d r x I R 1$ animals, indicating that there was no decrease in proteasome activity (Fig. 2d). To confirm that the reporter was sensitive to decreased proteasome activity, we reduced expression of the rpn-6.1 subunit of $19 \mathrm{~S}$ regulatory complex of the proteasome via RNAi [58] and detected an increase in Dendra2 fluorescence (Additional file: Fig. S3A). These data indicate that increased polyglutamine aggregation in the muscle cells of $d r x I R 1$ animals is not due to the changes in protein degradation or in polyQ protein levels.

Next, we tested two main transcriptional pathways known to regulate cytosolic protein homeostasis-insulin/IGF signaling and the heat-shock response. Increased activity of DAF-16/FOXO, the transcription factor of the insulin/IGF signaling pathway, is associated with improved proteostasis and has been shown to affect polyglutamine aggregation [30,36]. We found that neither genetic inactivation of daf-16, using daf-16(mu86) mutation [59], nor overexpression of active DAF-16::GFP protein [60] were able to revert the increased aggregation seen in $d r x I R 1$;Q40 animals (Fig. 2e). HSF-1/HSF1 is the heat-shock transcription factor that functions as a master regulator of molecular chaperones, degradation machinery, and other proteostasis components in the cytosol, and has also been shown to affect polyQ aggregation in wild-type animals [36]. Similarly to DAF-16, neither the hypomorphic hsf-1(sy441) allele, deficient in the heat-shock response [61], nor HSF-1 overexpression [62] were able to revert the increased aggregation caused by $d r x I R 1$ background (Fig. 2e). Together, these data indicate that the DR1350-derived variants in $d r x I R 1$ are not likely to act by modifying the basal proteostasis of the muscle cells of $C$. elegans.

\section{Variants in the introgressed interval do not alter biophysical properties of polyQ40 aggregates}

Besides changes in the cellular proteostasis of muscle cells, increased aggregation in $d r x I R 1 ; \mathrm{Q} 40$ animals could reflect changes in the amyloid-like nature and/or biophysical properties of polyQ40 aggregates themselves. PolyQ40 is known to form immobile aggregates that do not recover after photobleaching and are resistant to treatment with the detergent SDS [30,63]. Thus, we tested whether the presence of $d r x I R 1$ interval altered these properties of polyQ40 aggregates. As expected, photobleaching foci within Q40Bristol resulted in essentially no recovery of fluorescence, while soluble Q40:: YFP protein rapidly recovered to pre-bleach levels (Fig. 3a). We found no difference in recovery of Q40:: YFP foci between $d r x I R 1 ; \mathrm{Q} 40$ and Q40Bristol animals (Fig. 3a), indicating similarly immobile aggregates. To test for SDS resistance, we extracted aggregates from Q40Bristol and drxIR1;Q40 animals and treated them with 5\% SDS at room temperature, as described in [39]. We found polyQ aggregates to be similarly SDS resistant in both genetic backgrounds (Fig. 3b). To confirm that our SDS treatment could dissociate non-amyloid protein assemblies, we tested GFP::UNC-54 protein that forms myofilaments (as shown in Fig. 2b). Filamentous GFP:: UNC-54 protein was efficiently dissociated by SDS treatment in extracts from both Bristol and drxIR1 backgrounds (Fig. 3b).

Recently discovered positive regulator of aggregation, MOAG-4/SERF, which specifically distinguishes amyloid and non-amyloid aggregation [64, 65], was shown to affect Q40::YFP protein in C. elegans: decrease of moag4 expression via RNAi suppressed Q40 aggregation [65]. To test whether the variants in the $d r x I R 1$ background act through MOAG-4, expression of moag-4 was knocked down by RNAi in Q40Bristol and drxIR1;Q40 animals. moag-4 RNAi strongly decreased polyQ40 aggregation in both backgrounds, confirming the amyloidlike nature of aggregation in both (Fig. 3c (L4 animals) and Additional file: Fig. S3B (young adults)). However, drxIR1;Q40;moag-4(RNAi) animals retained higher aggregation relative to $\mathrm{Q} 40 \mathrm{Bristol}$;moag-4(RNAi) animals (Fig. 3c), as well as the increased susceptibility of the head muscles (Additional file: Fig. S3B), arguing against the $d r x I R 1$ interval variants acting through MOAG-4mediated mechanism. Together, our data suggest that neither decrease in muscle proteostasis nor changes in the aggregation pathway are responsible for the increased aggregation in $d r x I R 1 ; \mathrm{Q} 40$ animals.

\section{The increased aggregation is specific to polyglutamine expansions}

To determine whether the variants responsible for increasing polyQ40 aggregation in $d r x I R 1 ; \mathrm{Q} 40$ animals were acting generically on any amyloid aggregates, we asked if they can modify an aggregation-prone $A \beta$ peptide. We chose the muscle-specific $A \beta_{1-40}:: C F P$ transgene [66] because it exhibits both soluble and aggregated protein early in adulthood. We found that introduction of the $d r x I R 1$ interval did not increase $\mathrm{A} \beta$ aggregation (Fig. 3d). In contrast, when the $d r x I R 1$ locus was introduced into another polyglutamine model, Q35Bristol, we observed both the overall increase in 


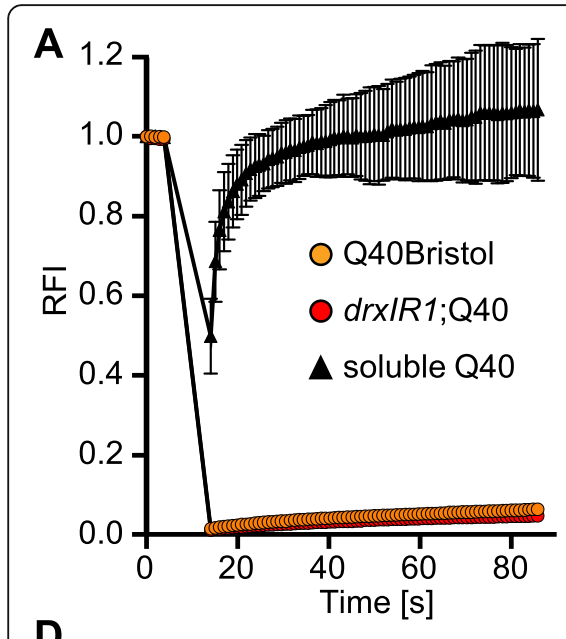

D



$A \beta_{1-40}:$ CFP
B

SDS: -

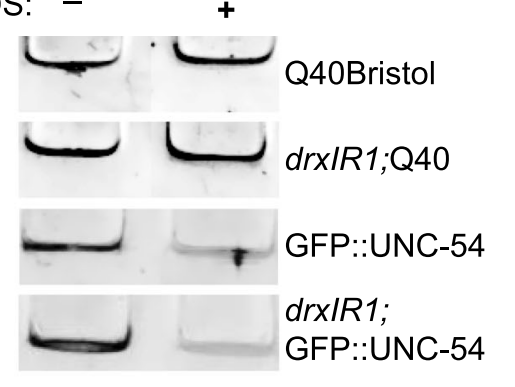

E

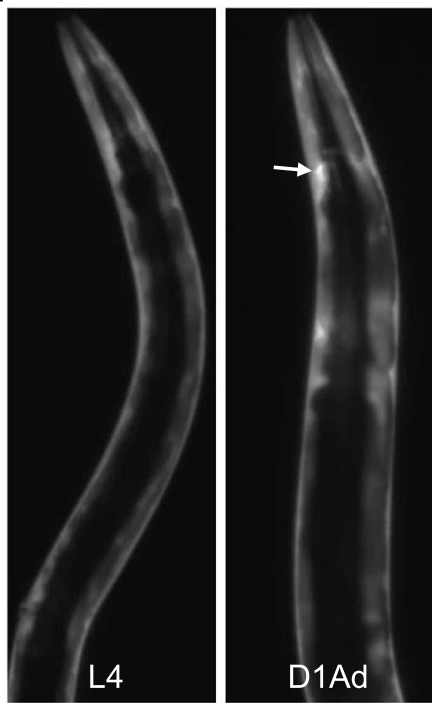

Q35Bristol

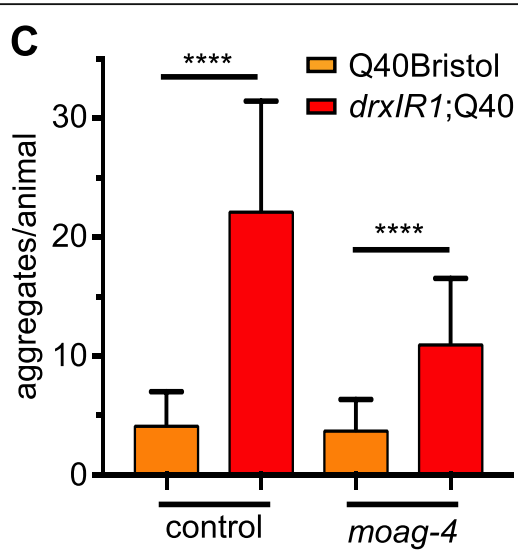

C

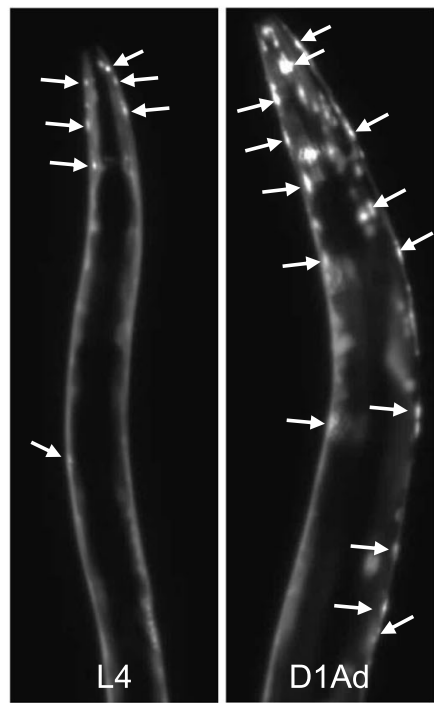

drxIR1;Q35

Fig. 3. Variants in $d r x I R 1$ interval do not alter the biophysical properties of polyQ aggregates. a FRAP analysis. The soluble Q40::YFP protein recovered rapidly (triangles), while aggregated protein (circles) in both Q40Bristol and drxIR1;Q40 backgrounds does not recover. Data are mean \pm SD. $\mathbf{b}$ PolyQ40 aggregates in native extract from drxIR1;Q40 animals remain resistant to 5\% SDS. Aggregated proteins fail to enter the native gel, remaining in the wells (shown). Native extracts containing the fibrillar GFP.:UNC-54 protein were used as controls. c The increased aggregation phenotype in animals carrying the drxIR1 interval does not depend on the amyloid-specific modifier moag-4 (mid-L4 animals; YA animals are shown in Suppl. Fig. 3B). Data are mean \pm SD, three independent experiments. Thirty-eight to 46 animals per condition. Data were


in unaffected by the $d r x I R 1$ locus. Shown are confocal stacks, arrows point to aggregates, and asterisks indicate $A \beta_{1-40:: C F P}$ accumulating in the nuclei of the muscle cells. Scale bar, $10 \mu \mathrm{m}$. e The shorter polyQ expansion (Q35::YFP) exhibits both the increased susceptibility of the head muscle cells and the accelerated overall aggregation in animals carrying the drxIR1 interval. Shown are stereo micrographs; arrows point to some of the aggregates. D1Ad, day 1 adults

polyQ35 aggregation and the increased susceptibility of the head muscles (Fig. 3e).

These data indicate that the DR1350-derived variants in $d r x I R 1$ background act by a polyglutaminespecific mechanism that is likely distinct from the known aggregation-modifying mechanisms. In addition, the effect on the Q35::YFP and Q40::YFP but not on $A \beta_{1-40}:$ CFP transgenic proteins confirms that the novel mechanism acts at the protein level, rather than by modifying the transgene genomic environment, since all three transgenes were made by the same approach.

Increased polyQ40 aggregation in the body-wall muscle cells and switch in susceptibility of the head muscles to aggregation are caused by genetically separable mechanisms

Since we were unable to narrow down the candidate genes by identifying affected pathways, and our data pointed to a potentially novel pathway, we turned to an 
unbiased investigation of genes in the interval. As we previously reported [38], the increased susceptibility of the head muscles to aggregation (RIL2-like phenotype, measured as the ratio of head to body aggregation) behaves as a recessive trait (Additional file: Table S1, top row), and is fully suppressed in $d r x I R 1$ heterozygous $(d r x I R 1 /+; Q 40)$ animals. Thus, we asked whether it was caused by a loss of function of a gene or genes in the interval, by testing whether it can be rescued in the drxIR1 homozygotes by introducing a wild-type copy of the interval. We used a free duplication $\mathrm{sDp} 2$ that covers the left arm of chromosome I, through $d p y-5$ gene in the center of the chromosome [46]. Introduction of sDP2 into animals homozygous for the $d r x I R 1$ interval and for the known loss-of-function $d p y$-5(e61) allele suppressed both the $d p y$ and the RIL2-like head phenotypes to the same extent (Additional file: Table S2, second row), indicating that the switch in head-muscle susceptibility phenotype in $d r x I R 1$ animals is caused by a loss-offunction variant(s), and therefore can potentially be identified by RNAi approach in Q40Bristol animals.

In contrast, the second polyQ phenotype, the increased overall aggregation (as scored in the body-wall muscles alone, excluding the head muscles), was not suppressed in animals heterozygous for the drxIR1 interval (Fig. 4a). Moreover, introduction of the sDP2 duplication, carrying the wild-type (Bristol) copy of this interval, into either Q40Bristol or drxIR1;Q40 animals resulted in sharply increased aggregation of polyQ40 in the body-wall muscles, relative to the corresponding strains without the duplication (Fig. 4a). This suggests that the phenotype of increased aggregation in the bodywall muscles depends on the dosage of a gene or genes within the boundaries of the modifier interval, and that in $d r x I R 1 ; \mathrm{Q} 40$ animals, this gene carries hypermorphic variant(s), mimicking increased gene dosage. Thus, the candidate gene may be identified by RNAi approach in drxIR1;Q40 animals.

\section{Autophagy-related gene 5 (ATG-5) is responsible for increased aggregation}

To decrease the number of genes that were to be tested by RNAi, we were able to further narrow the large drxIR1 interval (Additional file: Fig. S1B, C) to approximately $326 \mathrm{~Kb}$ (ChrI:1,647,221-1,972,719) by additionally backcrossing the $d r x I R 1 ; \mathrm{Q} 40$ animals and using the SNPs in the interval to detect recombination. The smaller $326 \mathrm{~Kb}$ interval contained 57 total genes including 25 candidate protein-coding genes with potentially functionally significant SNPs (based on SnpEff annotations [67], see the "Methods" section), with 24 candidate genes remaining after exclusion of egl-30 (Additional file: Table S2 and Additional file: Data File 1). Each of the candidate genes was knocked down by feeding RNAi in both Q40Bristol and $d r x I R 1 ; \mathrm{Q} 40$ animals, followed by quantification of polyQ aggregation.

None of the RNAi clones affected the increased susceptibility of the head muscles to polyQ aggregation (measured as a ratio of head to body aggregation) in either background. This may potentially indicate that more than one gene in the interval was responsible for the switch in the head muscle susceptibility, or that it depends on SNPs in non-coding RNAs, intergenic regions, or genes with SNPs that were not selected as potentially functionally significant; alternatively, this failure could be due to an inefficient knockdown. On the other hand, RNAi of several genes modified the second phenotype-the overall aggregation of polyQ40 in the body-wall muscle cells. Decreasing expression of two genes, Y71G12B.23 and C53H9.3, caused an increase in the number of aggregates in the Q40Bristol animals, with no change in the $d r x I R 1 ; \mathrm{Q} 40$ animals, while knocking down expression of atg-5 caused a large decrease in aggregation in the drxIR1;Q40 strain, with no effect in the Q40Bristol background (Fig. 4b). Because reversal of increased aggregation specifically in $d r x I R 1 ; \mathrm{Q} 40$ animals by RNAi is consistent with our genetic analysis for this phenotype in Fig. 4a, which suggested that the causative variant in drxIR1 background is hypermorphic, this points to atg-5 as a candidate gene. Based on the genome sequencing, atg-5 gene in $d_{r x I R 1 ; \mathrm{Q} 40}$ strain contains unique SNPs in its 3' UTR (Additional file: Data File 1).

The hypermorphic effect of SNPs localized in regulatory regions can be caused by increased expression of the affected gene or protein. qPCR data revealed no differences in atg-5 transcript levels in $d r x I R 1$ or $d r x I R 1$; Q40 animals compared to their respective Bristol strains (Fig. 4c). Thus, we asked whether decreasing the protein expression via a targeted deletion of atg-5 could reverse the increased polyQ aggregation in $d r x I R 1 ; \mathrm{Q} 40$ animals, as expected if the variants were hypermorphic. We used atg-5(bp484) allele, which has a mutation in a splice donor site of exon 1 disrupting the protein's expression or function $[68,69]$. We found that unlike animals that carried one DR1350-derived and one Bristol copy of the interval (drxIR1/+;Q40), which exhibit increased aggregation (Fig. 4a), drxIR1 heterozygous animals carrying the atg-5 mutation in the Bristol-derived copy (drxIR1) atg-5;Q40) completely lost the increased aggregation phenotype (Fig. 4d). These data suggest that increased levels of ATG-5 protein cause increased polyglutamine aggregation in the body-wall muscle cells.

\section{Activation of autophagy has divergent effects on polyQ aggregation in different tissues}

ATG-5 is an orthologue of the autophagic budding yeast protein ATG5 and of human ATG5. ATG-5 contributes to the initiation of autophagy by forming a complex with LGG-3/ATG12 and ATG-16/ATG16L1, which is recruited 


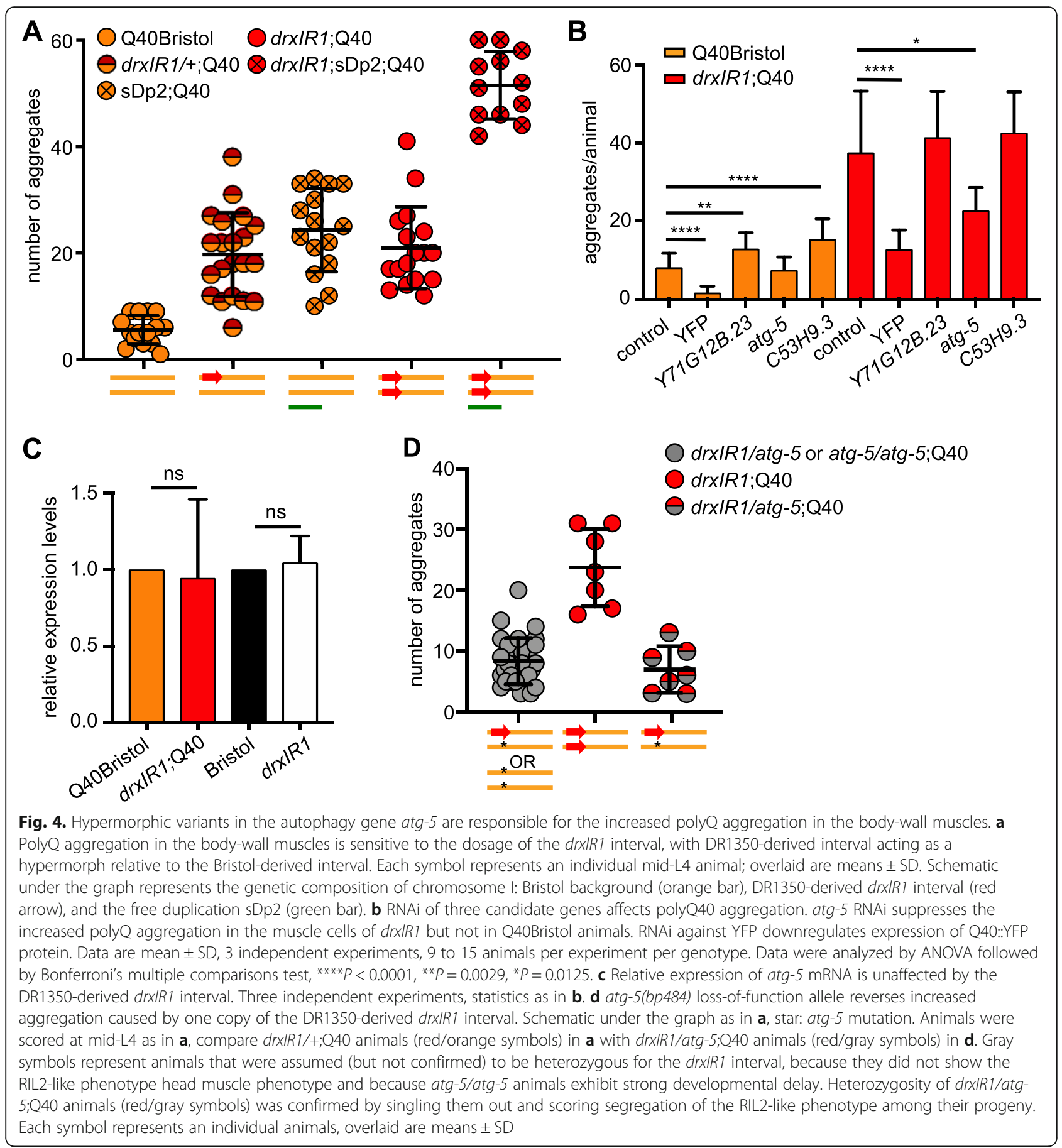

to the membrane of the elongating phagophore [70-72], and is required for the lipidation of LGG-1/LC3. Thus, upregulation or activation of ATG- 5 by the hypermorphic allele could cause either overactivation or an imbalance in autophagy. Interestingly, ATG5 in mammalian cells can also contribute to the progression of apoptosis, independent of its role in autophagy [73].

Although under basal conditions we saw no increase in the number of GFP::LGG-1 puncta in the muscle cells of $d r x I R 1$ animals (Fig. 2a), we did observe more puncta in the hypodermal cells, where autophagy is most readily induced in long-lived mutants [74]. Thus, we asked whether induction of autophagy the muscle cells was different in $d r x I R 1$ and wild-type (Bristol) animals under activation conditions. We used an autophagy inducer drug, ABT-737, that acts as a BH3-mimetic, inhibiting the antagonistic effects of Bcl-2 (CED-9 in worms) on Beclin-1 (BEC-1) and thus relieving inhibition of 
autophagy [75]. Treatment with $10 \mu \mathrm{M}$ of ABT-737 indeed induced GFP::LGG-1 puncta in the muscle cells of the wild-type (Bristol) animals (Fig. 5a). Surprisingly, animals carrying the $d r x I R 1$ interval exhibited an increase in punctate appearance of GFP::LGG-1 protein in the body-wall muscle cells already in response to the DMSO control. Although not previously reported to activate autophagy, low concentrations of DMSO have been reported to extend the lifespan of C. elegans and decrease the paralysis associated with $A \beta_{1-42}$ aggregation, when grown in liquid $[78,79]$. Importantly, ABT-737 resulted in a larger increase in GFP-positive puncta in drxIR1; GFP::LGG-1 animals compared to the Bristol background (Fig. 5a), indicating that $d r x I R 1$ interval increases accumulation of LGG-1/LC31-positive autophagosome structures in response to an activating treatment.

The larger increase in LGG-1 puncta in $d r x I R 1$;GFP:: LGG-1 animals could indicate that atg-5 hypermorphic allele causes either a stronger activation of autophagy or a slower lysosomal degradation. Because autophagy is known to promote clearance of polyglutamine aggregates [80], the increased aggregation in $d r x I R 1$ background appeared consistent with slower degradation, while activation of autophagy would have been expected to decrease aggregation [81]. To confirm this, we asked whether activation of autophagy with ABT-737 indeed decreased polyQ aggregation in the wild-type (Bristol) background. Surprisingly, treatment of Q40Bristol animals with this autophagy activator resulted in a large increase, rather than decrease, of polyQ40 aggregation in the body-wall muscles, with ABT-737-treated animals exhibiting a $44 \%$ increase in the number of aggregates (Fig. 5b). These data suggest that counter to expectations, activation of autophagy may enhance polyglutamine aggregation. We did not detect a further increase in aggregation in drxIR1 background, since the drug treatment protocol dictated scoring aggregates in young adult animals (see the "Methods" section), when aggre-

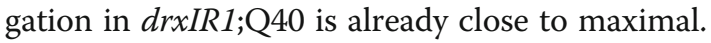

Because this effect of autophagy was unexpected, and because drug treatment may not be reliable in C. elegans, we tested two different genetic approaches known to activate autophagy to confirm these findings. Each of the two approaches activates autophagy via a mechanism distinct from that of ABT-737. First common approach is inactivation of mTOR [82]. In C. elegans, inactivation of LET-363/mTOR indeed activates autophagy, as shown by increase in GFP::LGG-1 puncta [83]. However, inactivation of LET-363 also causes larval arrest [84], which itself will affect polyQ aggregation. To overcome this, we targeted mTOR interacting protein MLST-8/mLST8, which is required for the kinase activity of mTOR [85], but can be downregulated in C. elegans without causing larval arrest [86]. RNAi knockdown of mlst-8 resulted in a 1.6-fold increase in polyQ40 aggregation in Q40Bristol animals (Fig. 5c, late-L4). Similar to the results of the drug treatment, mlst-8 RNAi had no significant effect in drxIR1;Q40 animals. We asked whether the apparent lack of effect on the $d r x I R 1 ; \mathrm{Q} 40$ animals was indeed due to the already high aggregate numbers at this developmental stage, by repeating the RNAi in younger animals, and observed an even stronger, 3-fold, increase in polyQ40 aggregation in Q40Bristol animals, and a 1.5fold increase in drxIR1;Q40 animals (Fig. 5c, mid-L4).

As a second genetic approach, we tested the effect of decreased activity of insulin/IGF-like signaling pathway, since reduction of function of the sole C. elegans orthologue of insulin/IGF receptor, DAF-2, is known to cause activation of autophagy, including in the body-wall muscle cells [57, 87]. Introduction of the hypomorphic daf-2(e1370) allele caused a 5.1-fold increase in aggregates in the Q40Bristol background, and 2.3-fold further increase in $d r x I R 1 ; \mathrm{Q} 40$ animals (Fig. $5 \mathrm{~d}$ ). The increase in polyQ aggregation in daf-2(e1370) background is consistent with previous reports [88]. Together, these pharmacological, RNAi, and genetic data suggest that aggregation of polyQ40 in the body-wall muscle cells is paradoxically increased by activation of autophagy.

Previous studies indicate that autophagy levels, both basally and in response to a trigger, can be different in different C. elegans and mammalian tissues [56, 89]. Intriguingly, in these reports, certain muscle groups in the mouse [89] and body-wall muscle cells in C. elegans $[56,57]$ exhibited lower basal autophagy compared to other tissues. Thus, we asked whether activation of autophagy may have a different effect on polyQ aggregation in muscles than in a different tissue. In addition to the muscle-expressed polyQs, the neuronal and intestinal fluorescent polyQ models have been established in C. elegans [76, 90]. We applied the same mlst-8 RNAi approach to the intestinal model and scored polyQ aggregation. Unlike in the muscle-expressing polyQ model, activation of autophagy via RNAi knockdown of $m l s t-8$ resulted in a large (3.5-fold) decrease in the percentage of animals exhibiting polyglutamine aggregation in intestine (Fig. 5e). Finally, we tested the effect of autophagy activation on polyglutamine aggregation in C. elegans neurons. The integrated polyQ67 expansions expressed at low levels from the pan-neuronal F25B3.3 promoter (Q67n:: CFP) presents with both soluble protein and aggregates in day 1 adult animals. Because neurons in C. elegans are refractory to feeding RNAi, we introduced the $d r x I R 1$ locus into Q67n::CFP animals, and scored the number of aggregates in the neurites of head neurons (Additional file: Fig. S4). Strikingly, we found that like in the intestine but unlike in the muscle cells, introduction of the drxIR1 locus into the neuronal polyQ model significantly decreased the number of CFP-positive aggregates in the neurites (Fig. $5 \mathrm{f}$ and Additional file: Fig. S4). Of 

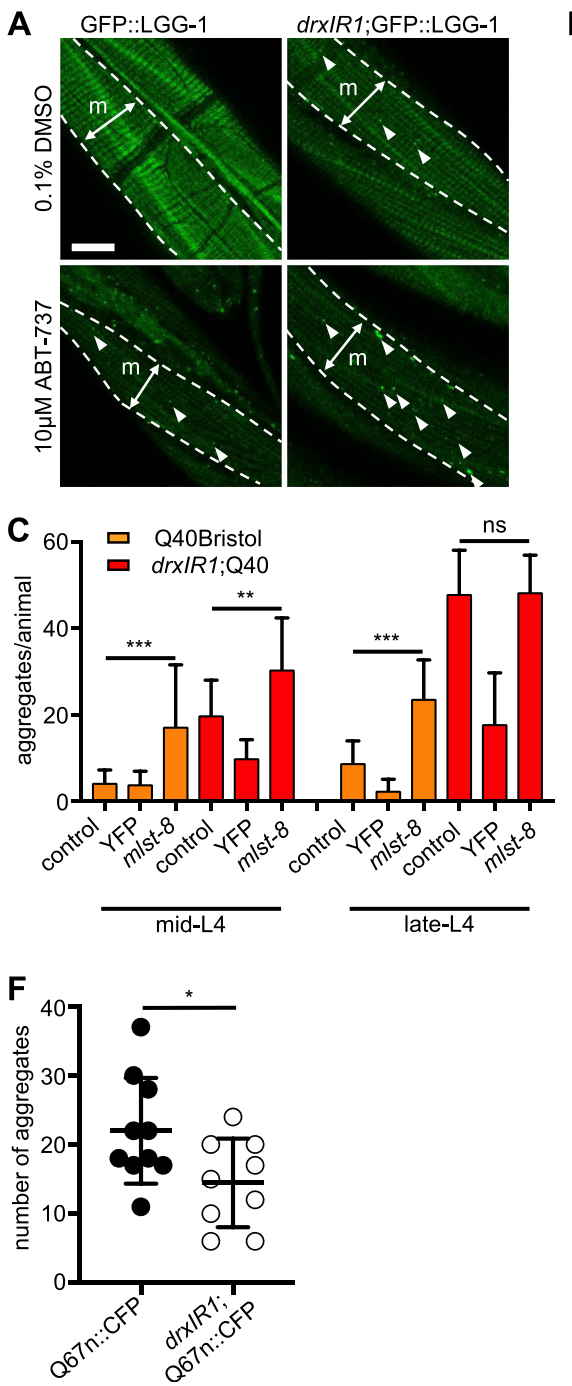
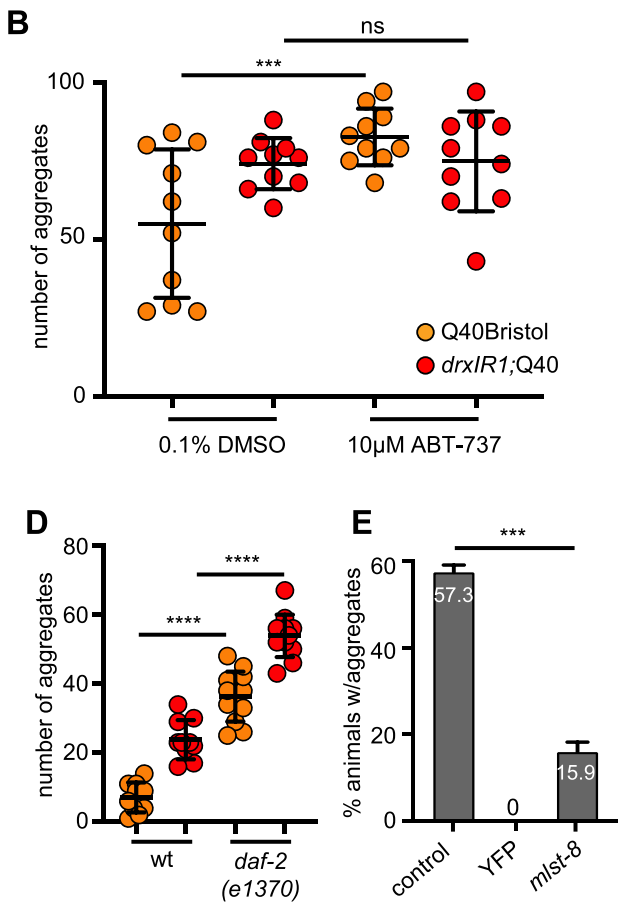

Fig. 5. Activation of autophagy has divergent effects on polyQ40 aggregate clearance in different tissues. a Animals carrying the $d r x \mid R 1$ interval accumulate more GFP::LGG-1-positive puncta (arrowheads) in the body-wall muscle cells upon treatment with autophagy-activating drug ABT737. Animals were treated with $0.1 \%$ DMSO (vehicle control) or $10 \mu \mathrm{M}$ ABT-737 for $24 \mathrm{~h}$. Shown are confocal projections; one muscle quadrant $(\mathrm{m})$ is indicated between punctate lines. Scale bar, $10 \mu \mathrm{m}$. b Autophagy-activating drug ABT-737 increases polyQ40 aggregation in the body-wall muscle cells in the wild-type background (Q40Bristol). Aggregation was scored in adult animals, 1 day post-L4 (see the "Methods" section).

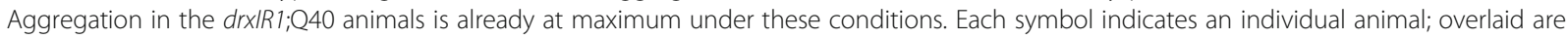
means \pm SD. Data were analyzed by ANOVA followed by Bonferroni's multiple comparisons test, ${ }^{* *} P=0.0006$. c Activation of autophagy with m/st-8 RNAi increases aggregation in the body-wall muscles of Q40Bristol mid- or late-L4 animals, and of drxIR1;Q40 mid-L4 animals. Data are mean \pm SD, 3 independent experiments, 9 to 13 animals per experiment per treatment. Control RNAi was mec-4. Data were analyzed by ANOVA followed by Bonferroni's multiple comparisons test, ${ }^{* *} P=0.0007,{ }^{* *} P=0.0082$. $\mathbf{d}$ Introduction of the daf-2(e1370) allele increases polyQ40 aggregation in the body-wall muscles in both Q40Bristol and drxIR1;Q40 animals. Aggregation was scored at mid-L4. Each symbol indicates an individual animal; overlaid are means \pm SD. Colors as in $\mathbf{b}$. Data were analyzed by ANOVA with Bonferroni's multiple comparisons test, ${ }^{* * * *} P<0.0001$. e Activation of autophagy with m/st-8 RNAi strongly suppresses polyQ aggregation in the intestinal cells. Percent of animals with Q44::YFP aggregates in the intestine of day 4 adult were scored, as in refs. [76, 77], for each indicated RNAi treatment. Control RNAi was mec-4. Data are mean \pm SD. Data were analyzed by ANOVA followed by Bonferroni's multiple comparisons test, ${ }^{* * *} P=0.0003$. $\mathbf{f}$ The $d r x I R 1$ interval decreases accumulation of polyQ67 aggregates in the neurites of head neurons. Aggregation was scored in day 1 adults over the dendritic area in the head, as shown in Additional file 4: Figure S4. Each symbol indicates an individual animal; overlaid are means \pm SD. Data were analyzed by an unpaired $t$ test, two-tailed, ${ }^{*} P=0.0332$

note, protein aggregation-induced trafficking defects in neurites are common in neurodegeneration, and autophagy in neurons is known to be regulated in a compartment-specific manner [91].
Together, these data show that depending on the tissue, activation of autophagy can either clear polyglutamine aggregates or increase their accumulation. 


\section{Discussion}

Using natural genetic variation, we identified an unexpected divergence in how activation of autophagy in different tissues impacts the behavior of aggregation-prone polyglutamine expansions. It is broadly appreciated that autophagy can be both protective and detrimental to cells and organisms [92]. For example, ER stress-induced autophagy is protective in cancer cells but contributes to apoptosis in non-transformed cells [93], while starvation-triggered autophagy in C. elegans pharyngeal muscle can switch from protective to pro-death, depending on its level of activation [55]. However, with respect to clearance of misfolded aggregated proteins, activation of autophagy is generally considered to be a positive, protective response [94, 95]. Therefore, activation of autophagy has been thought of as a nearly universal therapeutic approach to neurodegenerative diseases caused by protein aggregation [96]. The divergence in how polyQ expansions in neuronal, intestinal, and muscle cells respond to activation of autophagy suggests that interplay between autophagy and protein aggregation depends on the cellular context. We find that both the natural variants in atg-5, and the more traditional genetic and pharmacological ways of activating autophagy independent of atg-5, increased rather than decreased polyQ aggregation in the muscle cells of $C$. elegans. This represents a striking departure from the current paradigm. On the other hand, polyQ aggregation in neuronal and intestinal cells, as expected, was decreased by the same treatment. Considering the significant involvement of skeletal muscle in HD and other polyglutamine diseases, including the induction of the muscle catabolic phenotype and muscle wasting [97-101], a more nuanced understanding of integration of autophagy with cellular physiology is needed.

The use of natural variation was instrumental in uncovering this unexpected cell-specific effect of autophagy on protein aggregation. The DR1350-derived variants that we identified as being responsible for the increased aggregation of polyQ40 in the muscle cells are in the regulatory 3'UTR region of the atg-5 gene. Although 3' UTR variants could affect activity in some proteins, for example, by affecting localization of mRNA and thus its local translation, our genetic analysis points to the gain of expression as the mechanism of atg-5 variants. Based on the ability of one additional copy of the wild-type, Bristol-derived atg-5 to mimic the effect of these natural variants (Fig. 4a), and because deletion of one copy of atg-5 reverses the effect of the DR1350-derived variants in the remaining copy (Fig. $4 \mathrm{~d}$ ), we estimate that the variants increase the expression of ATG-5 protein by less than twofold. Strikingly, introduction of one additional Bristol-derived copy of atg-5 into the animals already carrying two DR1350-derived hypermorphic alleles increases the polyQ aggregation even further, to about sixfold above normal. This indicates a quantitative relationship between the levels of ATG-5 protein and increased polyQ aggregation in the muscle. Although we are currently unable to directly modulate autophagy in C. elegans in a graded manner, the ability of three distinct methods of activating autophagy to mimic the effect of the variants argues that the increase in ATG-5 affects the polyQ aggregation by increasing autophagy, rather than for example by causing stoichiometric imbalance and autophagy inhibition [81], or coupling to apoptosis pathway [73]. The precise mechanistic basis of this quantitative relationship will need to be investigated further.

Our approach in identifying the modifier variants was different from the traditional QTL mapping and was modeled on the EMS-density mapping method for mutation identification [102]. We consider that the serial backcrossing/selection scheme we used prior to whole genome sequencing provides a generalizable approach to mapping modifier variants in C. elegans, as it allows for rapid enrichment of causative variants even from a single available modifier background. This method also simultaneously tests whether putative modifiers work in different genetic backgrounds. Finally, because multiple pathways can impinge on protein homeostasis, and in addition, weakly-destabilizing coding polymorphisms across genome can have strong effects on protein aggregation or toxicity [37], each modifier background may contain multiple loci contributing a combined effect. The serial backcrossing/selection scheme tolerates selecting of only a small number of recombinant animals at each backcross, or even a singular animal exhibiting the desired phenotype, and thus may be preferable for such multi-loci modifiers.

One important aspect of our findings is the cryptic nature of the modifier variants in atg-5. Cryptic variation typically does not cause phenotypic changes on its own, but becomes phenotypically "exposed" when challenged with a stressful environment, thus contributing to disease susceptibility [103-105]. Polyglutamine expansions may mimic cellular stress, for example, by destabilizing the folding environment [37] or disrupting transcriptional control [106]. Indeed, the atg-5 variants identified here as modifiers are derived from a phenotypically normal wild strain DR1350, and we did not detect significant alterations in the basal autophagy in the muscles of $d r x I R 1$ animals, until they were challenged with the aggregation-prone polyQ40, or with autophagyactivating drug ABT-737.

In addition to being exposed by stress, the phenotypic expression of cryptic modifier variants may reflect their more direct interactions with the disease-causing mutation. For example, in humans, analysis of HD modifier 
loci on chromosomes 8 and 15 showed that these variants influence certain clinical readouts in subjects with expanded polyQ tracts, prior to the appearance of disease symptoms, while they have no major effects in control individuals without expansions [18]. The suspected culprit for the modifying effect of the chromosome 15 locus, the DNA endo/exonuclease FAN1, may be changing the disease phenotypes or age of onset by directly affecting the stability of the polyQ-encoding repeat in somatic tissues $[18,107]$. Interestingly, that study also suggested that modifiers could have distinct effects in different cell populations.

In our study, the cryptic nature of the atg-5 variants allowed detection of the unusual tissue dependence of the relationship between autophagy and aggregation, because stronger variants which ectopically activate autophagy already under basal conditions often have additional strong phenotypes potentially masking changes in polyQ aggregation. For example, loss of function of C. elegans mTOR leads to larval arrest [84], while hypomorphic mutations in insulin/IGF signaling pathway, in addition to activating autophagy, trigger numerous other developmental, stress responsive, and metabolic pathways [108-110]; both can thus have their own effects on the aggregation-prone protein. Even nongenetic means such as activation of autophagy by nutrient deprivation are accompanied by the metabolic and protein expression changes [111] that can mask the more specific effect on the polyQ behavior. Natural variation may thus indeed identify the candidate modifier pathways and mechanistic relationships in aggregation diseases that are distinct from those identified by the traditional approaches.

The reasons the muscle cells are differentially sensitive to autophagy with respect to protein aggregation, or why this is not true for other aggregation-prone proteins, are not yet known. The selectivity towards the polyglutamine expansions would argue against a global dysregulation of protein homeostasis in the muscle cells of $d r x I R$ lanimals, which is supported by our data. It is possible, however, that ectopic activation of autophagy disrupts select proteostasis processes that only impinge on the polyQ aggregation or clearance in these cells. Another possibility is that autophagic degradation of polyQ expansions requires a specific "signal" or adaptor, which may be competed away during general increase in autophagy in the muscle cells, but remains sufficient in intestine or neurons. The polyQ-expanded huntingtin protein $(\mathrm{Htt})$ indeed requires specific adaptors, such as Tollip, to be cleared by autophagy [112], although whether this is also true for polyQ expansions outside the Htt context is not clear. Yet another possibility is that polyQ expansions themselves interfere with autophagy. For example, polyQ-expanded $\mathrm{Htt}$ have been suggested to interfere with the delivery of cargoes to autophagic vacuoles [113], and shown to co-aggregate with the autophagy adaptor Tollip, potentially disrupting other functions of this multi-tasking protein [112]. If so, the low basal levels of autophagy may render the proteostasis of the muscle cells to be selectively sensitive to the polyQ expansions.

Muscle cells may also have a different regulation of or dependence on autophagy, because autophagy of the muscle is an adaptive response of many metazoans to starvation [114]. While basal autophagy is important for muscle maintenance, its over-activation can lead to muscle atrophy [115-117]. Indeed, in C. elegans, bodywall muscles in young animals have low basal levels of autophagy relative to other tissues [56, 57], while in mice, the slow-twitching (soleus) muscles exhibited little induction of autophagy after $24 \mathrm{~h}$ of starvation, as defined by the autophagosome counts, distinct from the fast-twitching (extensor digitorum longus) muscles that had significant induction [89]. Moreover, the distribution of autophagosomes was different between the fastand slow-twitching muscle types, supporting the idea of differential autophagy regulation in different cells or tissues.

In addition to the traditional mouse models, the genetic model systems such as worm, fly, and yeast, in which natural variation can be readily combined with modeling the gain-of-function disease mutations by transgenesis, offer new opportunities to identify the cryptic modifier pathways for neurodegenerative and protein aggregation diseases [10, 118-122]. Examples of this approach include a study with Drosophila Genetic Reference Panel [123] that uncovered an unexpected role of heparin sulfate protein modifications in modifying the toxic effects of the misfolded mutant of human insulin, a cause of permanent neonatal diabetes [124], and a recent study in C. elegans that showed that the ability of $\alpha$-synuclein to cause transcriptional and phenotypic changes is substantially modified by the genetic background [40]. The important feature of the cryptic modifier pathways that can be identified by these approaches is that they harbor natural variants shaped by selection, and thus will pinpoint the naturally plastic potential genes and networks [14], amenable to pharmacological manipulation without negative effects on the organism.

\section{Conclusion}

Our work identifies a divergence in the ability of autophagy to clear aggregates in different tissues. As activation of autophagy is a promising therapeutic strategy for protein aggregation diseases, the vulnerability of muscle cells in our study highlights the need for a more nuanced understanding of how autophagy integrates with cellular physiology. 
Importantly, the finding that dramatic differences in polyglutamine aggregation can be caused by physiologicallevel differences in the autophagic response, encoded in wild-type genomes, supports the use of natural genetic variation in model organisms to interrogate pathways that confer protection or susceptibility in protein aggregation diseases.

\section{Methods}

\section{Nematode strains and growth conditions}

Nematodes were grown at $20^{\circ} \mathrm{C}$ on nematode growth medium (NGM) plates, seeded with E. coli OP50 [125]. Animals were synchronized by picking gastrula stage embryos onto fresh plates, unless otherwise noted.

The following stains were obtained from Caenorhabditis Genetics Center (CGC): AM141 [rmIs333(punc-54:: Q40::YFP)], AM140 [rmIs132(punc-54::Q35::YFP) I], CF1038 [daf-16(mu86) I], TJ356 [zIs356(pdaf-16::daf16a/b::GFP;pRF4(rol-6(su1006)) IV], PS3551 [hsf1(sy441) I], DA2123 [adIs2122(plgg-1::GFP::lgg-1 + rol6(su1006))], KR1108 [unc-11(e47) dpy-5(e61) I], KR292 [him-1(h55) dpy-5(e61) unc-13(e450) I; sDp2 (I;f)], MT1434 [egl-30(n686) I], CB1370 [daf-2(e1370) III], and AM44 [rmIs190(pF25B3.3::Q67::CFP)]. TGF205 [xzEx3(punc-54::UbG76V::Dendra2)] was made by crossing out $g l p-1(e 2141)$ from AGD1033.

The AS408 [punc-54::GFP::UNC-54], AM583 [rmIs249(plet-858::hsf-1;pmyo-2::GFP)], AM738 [rmIs297(pvha-6:: Q44::YFP;rol-6(su1006))], and AM930 [rmIs335(punc-54:: A $\beta$ (1-40)::CFP)] strains were kindly provided by the Morimoto lab, and the HZ1732 [atg-5(bp484) I; him-5] strain by the Colón-Ramos lab. The Q40DR1350 and recombinant inbred lines (RILs) 2, 12, 12(2), and 15 were described in [38].

The drxIR1(I, DR1350>Bristol) locus and/or the Q40 locus was introduced by genetic crosses into the following strains: TGF134 [drxIR1;rmIs333(punc-54::Q40::YFP)], TGF130 [drxIR1;punc-54::GFP::UNC-54], TGF353 [drxIR1; adIs2122(plgg-1::GFP::lgg-1 + rol-6(su1006))], TGF208 [xzE x3(punc-54::UbG76V::Dendra2);rmIs333(punc-54::Q40::YFP )], TGF207 [drxIR1;xzEx3(punc-54::UbG76V::Dendra2);rmI s333(punc-54::Q40::YFP)], TGF088 [daf-16(mu86) I; rmIs 333(punc-54::Q40::YFP)], TGF188 [drxIR1;daf-16(mu86) I; rmIs333(punc-54::Q40::YFP)], TGF086 [zIs356(pdaf-16::daf16a/b::GFP;pRF4(rol-6(su1006)) IV;rmIs333(punc-54::Q40:: YFP)], TGF190 [drxIR1;zIs356(pdaf-16::daf-16a/b::GFP; pRF4(rol-6(su1006)) IV;rmIs333(punc-54::Q40::YFP)], TGF 187 [hsf-1(sy441) I;rmIs333(punc-54::Q40::YFP)], TGF170 [drxIR1;hsf-1(sy441) I;rmIs333(punc-54::Q40::YFP)], TGF 036 [rmIs249(plet-858::hsf-1;pmyo-2::GFP);rmIs333(punc54::Q40::YFP)], TGF189 [drxIR1; rmIs249(plet-858::hsf-1; pmyo-2::GFP);rmIs333(punc-54::Q40::YFP)], TGF203 [drxIR 1;rmIs335(punc-54::A $\quad(1-40)::$ CFP)], TGF342 [drxIR1; rmIs132(punc-54::Q35::YFP) I], TGF261 [rmIs333(punc-54::
Q40::YFP);him-1(h55) dpy-5(e61) unc-13(e450) I; sDp2 (I; f)], TGF275 [drxIR1;rmIs333(punc-54::Q40::YFP); him1(h55) dpy-5(e61) unc-13(e450) I; sDp2 (I;f)], TGF089 [daf2(e1370) III;rmIs333(punc-54::Q40::YFP)], and TGF127 [drxIR1;daf-2(e1370) III;rmIs333(punc-54::Q40::YFP)].

The $\operatorname{drxIR1;Q40}$ strain was made by the following scheme: Q40Bristol males were mated to RIL2 hermaphrodites, and 5-10 F1 hermaphrodite progeny, identified by the lack of RIL2-like increased head aggregation phenotype, were picked onto fresh plates. F2 generation was examined for the expected 1:3 segregation of the increased head aggregation phenotype, and 7-10 F2 hermaphrodites with this phenotype were further mated with Q40Bristol males. This mating-selection cycle was repeated 23 times. The resulting strain was named drxIR1;Q40.

The introduction of $d r x I R 1$ locus by genetic crosses was confirmed by detecting the presence of the SNP 5 (WBVar00016276) (Additional file: Fig. S1C): a 743-bp fragment containing the variant was amplified using the drxIR1 primers (Additional file: Table S3), at an annealing temperature of $60{ }^{\circ} \mathrm{C}$, to produce an amplicon of $743 \mathrm{bp}$, and the PCR product was digested with SalI. The SalI site is present in the Bristol background, producing $432 \mathrm{bp}$ and $311 \mathrm{bp}$ products after the digest, but is absent in the DR1350 background.

\section{Genome sequencing}

The $23 \times$ backcrossed RIL2 strain, renamed as $d r x I R 1$; Q40, and the Q40Bristol stock that was used as the parental strain during backcrossing procedure were collected for sequencing within 2-3 generations after the last backcross. Strains were also cryopreserved at this time. A total of 20-30 animals of each strain were allowed to propagate on several $10-\mathrm{cm}$ plates seeded with OP50 bacteria. Upon depletion of the bacteria, animals were collected, washed, and flash frozen for genomic DNA extraction. Genomic DNA from $d r x I R 1 ; Q 40$ and Q40Bristol frozen pellets was extracted using phenol:chloroform (Sigma, USA). DNA was sequenced using the NextSeq 500 System (Illumina, USA) at the Wistar Institute (Philadelphia, PA, USA). Unpaired short reads were analyzed using the Galaxy [126] CloudMap pipeline, as described in [47], against WS220 genome assembly. Variants identified in the genome of Q40Bristol strain, which was used for serial backcrossing of RIL2 to generate the $d r x I R 1 ; Q 40$ strain, were subtracted from the $d r x I R 1 ; \mathrm{Q} 40$ SNPs. Because Hawaiian background did not previously cause increased polyQ aggregation [38], we also subtracted the known Hawaiian variants, using the Hawaiian SNP file within the CloudMap pipeline. This likely did not remove all the variants that overlapped between the Hawaiian and $d r x I R 1 ; \mathrm{Q} 40$ background, as the file did not contain the additional 
variants identified in ref. [48]. Finally, the CloudMap SnpEff tool was utilized to annotate the resulting genetic variants and predict their functional effects on genes and proteins [67]. SNPs with the following annotations were considered as potentially functionally significant: nonsynonymous coding, start gained or lost, stop gained or lost, splice site donor/acceptor, frameshift, and $5^{\prime}$ or $3^{\prime}$ UTR.

\section{Quantification of polyQ40 aggregation}

Aggregation was scored by counting fluorescent foci in images collected from animals immobilized with $20 \mathrm{mM}$ $\mathrm{NaN}_{3}$. For aggregation in the muscle cells, images were obtained using a Leica M205FA stereoscope with a digital camera (Hamamatsu Orca R2). For synchronization, 1520 well-fed L4 animals from non-crowded plates were transferred to new plates, gastrula stage embryos were picked 2-3 days later, and hatched animals were allowed to develop for specified time or to specified developmental stage. Aggregation was scored in late-L4 animals, unless otherwise indicated. The developmental larval stage was confirmed based on the germline development, or by days since L4 (for older adults). For data expressed as means, the number of animals for each data point is indicated in the figure legends.

For aggregation in neurons, images were obtained by confocal microscopy, as described below. Confocal stacks were collapsed as maximum-intensity projections in ImageJ [127], and the number of aggregates was counted in the dendritic area of animal's head, as shown in Additional file: Fig. S4. This area contains mainly dendrites of sensory neurons, with some interneuron processes and few cell bodies and/or neurites of other types of neurons (https://www.wormatlas.org/neuronsandcir cuits.html). A total of 9-10 day 1 adult animals were scored per genotype.

\section{Microscopy}

For confocal images, animals were immobilized on 2\% agar pads with $20 \mathrm{mM} \mathrm{NaN}{ }_{3}$ and imaged with Zeiss LSM700 microscope at Cell Imaging Center, Drexel University. Z-stacks were acquired at $0.4 \mu \mathrm{m}$ intervals as 12 bit images, using $63 \times 1.4 \mathrm{NA}$ objective, and analyzed with ImageJ [127].

For the quantification of autophagic vesicles, Z-stacks were collapsed as maximum intensity projections, the muscle cells were outlined, and the GFP::LGG-1-positive puncta within the outlined cells were counted. Thirty to 40 cells from 8 to $10 \mathrm{~L} 4$ animals were analyzed per genotype. To compare GFP::UNC-54 protein levels, GFP fluorescence was measured within the same size area $(\sim$ $9 \mu \mathrm{m}^{2}$ ) in the center of each analyzed muscle cell, over the myofilaments. Sixteen to 20 cells from 4 to 5 animals per genotype were measured. An identical size area measured away from the myofilaments was used for background subtraction.

Fluorescent recovery after photobleaching (FRAP) was performed on day 2 adults (for aggregated Q40) and L4 larvae (for soluble Q40) animals, as in [90], using the Zeiss LSM700 confocal microscope. Photobleaching was performed with $488 \mathrm{~nm}$ laser, by 100 iterations at $100 \%$ laser power. Imaging during recovery was at $0.3 \%$ power. Relative fluorescence intensity (RFI) was determined with the following equation: $\mathrm{RFI}=\left(T_{t} / C_{t}\right) /\left(T_{0} / C_{0}\right)$, with $T_{0}$ representing the total intensity of the region of interest before photobleaching and $T_{t}$ the intensity in the same area at any time after. We normalized against an unbleached area in the same cell, where $C_{0}$ is a control area before bleaching and $C_{t}$ represents any time after bleaching [90]. Seven to 18 aggregates from 3 animals each were measured per strain for aggregated Q40, and 5 cells from 2 animals each were measured per strain for the soluble Q40 controls.

For stereo images, animals were immobilized on NGM plates in a drop of $20 \mathrm{mM} \mathrm{NaN}$. Imaging was performed using a Leica M205FA stereo microscope with an Orca R2 digital camera (Hamamatsu). The magnification and the intensity of fluorescent sources (Chroma PhotoFluor 2) were kept constant within experiments. UbG76V::Dendra2 animals were imaged with a narrowbandpass CFP filter (Chroma), to avoid the spectral overlap with the Q40::YFP protein.

\section{Native protein extracts}

To prepare native protein extracts, synchronized embryos were isolated by hypochlorite treatments and larvae were collected once they reached the L3 stage. Worms were washed and allowed to settle on ice, and the packed pellets were flash frozen after removal of supernatant. The worm pellets were mechanically disrupted and lysed in $0.5 \%$ Triton-X 100 buffer as described in [39]. For SDS solubility, native protein extracts were incubated in 5\% SDS for $15 \mathrm{~min}$ at room temperature prior to running on a $5 \%$ continuous native gel, at $25 \mathrm{mg}$ of total protein per lane. Gels were imaged on a Typhoon FLA7000 scanner (General Electric, USA) with ImageQuant TL software to quantify YFP fluorescence. All experiments were performed three times.

\section{qPCR}

Approximately $50 \mu \mathrm{l}$ pellets of $\mathrm{L} 4$ stage worms were flash frozen in liquid nitrogen, and RNA extraction was performed using TRIzol (Life Technologies, USA) and chloroform (Sigma, USA) reagents. The samples were treated with DNase (DNA-free, Life Technologies, USA) to remove any genomic DNA, and iScript cDNA synthesis kit (Bio-Rad, USA) was used to reverse transcribe 1$2 \mu \mathrm{g}$ of RNA per sample. The expression of selected 
genes was measured using iTaq Universal SYBR Green Supermix (Bio-Rad) and the ViiA detector (Applied Biosystems). Each biological replicate was run in triplicate, and data analyzed using the $\Delta \Delta C T$ method [128]. Three biological replicates were used to assess statistical significance. Gamma-tubulin ( $t b g-1)$ was used for normalization, as it was stable between the $d r x I R 1$ and the Bristol strains. Sequences for $t b g-1$ primers were as in ref. [129]. Primer sequences are listed in Additional file: Table S3.

\section{RNAi experiments and constructs}

For RNAi experiments, NGM plates containing $100 \mu \mathrm{g} /$ $\mathrm{ml}$ ampicillin and $0.4 \mathrm{mM}$ IPTG were seeded with control (L4440 empty vector, unless otherwise noted) or experimental overnight RNAi bacterial cultures and incubated at room temperature for 2 days prior to use. Worms were cultured on the RNAi plates from gastrula stage embryos for two generations. RNAi clones were from the Ahringer library (J. Ahringer, University of Cambridge, Cambridge, UK), except for those corresponding to mab-20, Y71G12B.18, Y71G12B.33, Y71G12B.23, Y71G12B.35, drag-1, Y71G12B.31, ubc-3, tln-1, Y71G12B.25, pghm-1, C53H9.3, tag-96, tub-2, Y51F10.4, and spe-48; these were made by cloning a unique 0.8 to $1.2 \mathrm{~Kb}$ fragment from each gene into the L4440 plasmid and transforming into the $E$. coli strain HT115. Primer sequences are listed in Additional file: Table S4. All experiments were repeated three times; the total (combined) number of animals is indicated in figure legends.

\section{ABT-737 treatment}

Twenty to 40 gastrula stage embryos were grown on OP50 bacteria for 2 days at $20^{\circ} \mathrm{C}$; nematodes collected, washed, and exposed to either 0.1\% DMSO (Sigma, USA) as solvent control, or $10 \mu \mathrm{M}$ ABT-737 (ApexBio, Taiwan). Earlier exposure to ABT-737 resulted in larval arrest. Animals were incubated in the drug solution with shaking for $24 \mathrm{~h}$, pipetted onto plates, and either scored for aggregation or imaged.

\section{Statistical analyses}

ANOVA and $t$ test analyses were performed with Prism software (GraphPad, USA), using $\alpha$ value of 0.5 . ANOVA was followed by Bonferroni's multiple comparisons post-test. All $P$ values and significance levels are indicated in the figures and figure legends.

\section{Supplementary information}

Supplementary information accompanies this paper at https://doi.org/10. 1186/s12915-020-0750-5.

Additional file 1: Figure S1. Schematic of the drxlR1 interval and SNPS used for mapping. (A) Red: the $1.4 \mathrm{Mb}$ genomic region on chromosome
I, containing the DR1350-derived intervals, in the RIL2-derived drxIR1:Q40 strain and the four remaining high aggregation RILS (RIL12, RIL12(2), RIL18 and RIL15); orange: the Bristol background. Punctate lines delineate the narrowed $326 \mathrm{~Kb}$ interval containing the candidate genes tested by RNAi. Diamonds: SNPs used to test for the presence of the interval; SNP 6b (Chrl:1,972,719 (WBVar00017376)) is Bristol-derived in drXIR1;Q40 and RIL15 animals. Locations of egl-30, moag-4 and the incompatibility locus zeel-1/peel-1are also indicated. The coordinates here correspond to the WormBase release WS270 [131]. (B) WormBase names and chromosomal locations of SNPs marked with diamonds in A.

Additional file 2: Figure S2. Cumulative distribution of unique SNPS across remaining chromosomes. chromosomes II through $\mathrm{X}$ in the drxIR1;Q40 strain accumulated up to 160 unique SNPs each. Shown are SNPs remaining after subtraction of the variants present in Q40Bristol strain, and of variants in the Hawaiian isolate that does not exhibit increased polyQ40 aggregation.

Additional file 3: Figure S3. Controls for the basal proteostasis effects of the drxIR1 locus. (A) The UbG76V:Dendra2 proteasome reporter is sensitive to decreased proteasome levels. Knockdown of a proteasome subunit rpn-6.1, via RNAi, increased the average intensity of the Dendra2 compared to control treatment. Images were taken and quantified as in Fig. 2a. Data are mean \pm SD. Data were analyzed by unpaired $t$-test, twotailed, ${ }^{*} P=0.0244$. (B) Stereomicrographs of young adult animals after treatment with control or moag-4 RNAi. moag-4 RNAi decreased aggregation in both backgrounds, but preserved the increased aggregation drxIR1;Q40 animals relative to Q40Bristol.

Additional file 4: Figure S4. The $d r x \mid R 1$ interval decreases accumulation of polyQ67 aggregates in the neurites. (A) Outline of the head of an individual animal, with intestine (int) and pharynx (ph) indicated. Anterior to the left, dorsal up. Green shows projection of Q67n::CFP fluorescence signal overlaid on the transmitted light image. Punctate line in the middle immediately below the anterior pharyngeal bulb separates the dendritic region where aggregation was scored (anterior to the line) from the area with many neuronal cell bodies and the axonal bundle. The scored region contains mainly dendrites of sensory neurons, with some interneuron processes and some cell bodies and/or neurites of other types of neurons (https://www.wormatlas.org/neuronsandcircuits.html). Scale bar is $20 \mu \mathrm{m}$. Same animal as in panel B, top. (B) Confocal images of heads of two Q67n::CFP animals in Bristol background, rotated into horizontal positions. Regions scored in Fig $5 f$ are anterior (to the left) to the punctate line (marked as dendrites). Examples of aggregates within the neurites are indicated by arrowheads. (C) Confocal images of drxIR1;Q67n::CFP animals. Labeling as in panel B. Areas marked as cell bodies and axons did not exhibit decreased aggregation in drxIR1 background relative to Bristol (compare to the same area in panel B). (D, E) Same images as in panels B and C, respectively, shown with enhanced fluorescence.

Additional file 5: Table S1. Loss-of-function analysis for the RIL2-like head aggregation phenotype. SDP2 free duplication covers most of the left arm of chromosome I, extending through dpy-5 marker but not through unc-13. drxIR1;Q40 animals were crossed with KR292 [him1(h55);dpy-5(e61);unc-13(e450)l; sDp2(l;f)], F1 progeny that either did (based on segregation of unc non-dpy phenotype among their progeny) or did not inherit the sDp2 duplication were singled, and their F2 progeny scored for the increased ratio of head to body aggregation (RIL2-like) and the dumpy phenotypes. The RIL2-like phenotype behaved genetically as did the known loss-of-function dpy-5(e61) allele.

Additional file 6: Table S2. Candidate genes tested by RNAi. 24 candidate genes present in the target $326 \mathrm{~Kb}$ of drxIR1 interval (between SNPs 5 and 6b (Additional file 1: Fig. S1)) are indicated in color. Genes were defined as candidates based on the SnpEff annotations (see Methods and Additional file: Data File 1). egl-30 was excluded based on genetic crosses. Genes in purple were targeted by clones from the Ahringer RNAi library. RNAi targeting constructs for genes in red were prepared in this work.

Additional file 7: Table S3. Primers used for genotyping the $d r x \mid R 7$ locus and for qPCR analysis of atg-5 expression.

Additional file 8: Table S4. Primers used for generating RNAi clones. 
Additional file 9: Data File 1. List of genes in the $d r x \mid R 1$ interval with potentially significant SNPs generated by the SnpEff tool. The nucleotide positions correspond to the N2(Bristol) genome assembly from WormBase release WS220 [131], available in the UCSC Genome Browser as ce10. The presence of human orthologs is according to [132].

\section{Acknowledgements}

We thank the Colón-Ramos lab at Yale School of Medicine and the Morimoto lab at Northwestern University for contributing worm strains, and Dr. David Raizen (University of Pennsylvania School of Medicine) for experimental suggestions. Some strains were provided by the Caenorhabditis Genetics Center (CGC) at the University of Minnesota, which is funded by $\mathrm{NIH}$ Office of Research Infrastructure Programs (P40OD010440). We would like to thank Anna Lysenko for experimental assistance.

\section{Authors' contributions}

JA-F contributed to the design of the work; the acquisition, analysis, and interpretation of data; and the writing of the manuscript. SH contributed to the acquisition, analysis, and interpretation of data, and writing of the manuscript. MY contributed to the acquisition and analysis of data. AAE contributed to the acquisition and analysis of data. CJ contributed to the acquisition and analysis of data. MV contributed to the design of the work TG contributed to the conception and design of the work, the analysis and interpretation of data, and the writing of the manuscript. All authors read and approved the final manuscript.

\section{Funding}

This study was supported by the NIH R36AG045411 grant. The funding agency had no role in the design of the study and collection, analysis, and interpretation of data.

\section{Availability of data and materials}

All data generated or analyzed during this study are included in this published article (and its additional information files). Strains are available from corresponding author on request. Sequencing data [130] is available in the NCBI repository, https://www.ncbi.nlm.nih.gov/sra/PRJNA598355.

\section{Ethics approval and consent to participate}

Not applicable

\section{Consent for publication}

Not applicable

\section{Competing interests}

The authors declare that they have no competing interests.

\section{Author details}

'Biology Department, Drexel University, Philadelphia, PA 19104, USA. ${ }^{2}$ Present Address: Department of Microbiology, Perelman School of Medicine, University of Pennsylvania, Philadelphia, PA 19104, USA. ${ }^{3}$ Department of Pathology and Laboratory Medicine, Children's Hospital of Philadelphia, Philadelphia, PA 19104, USA. ${ }^{4}$ Current Address: Department of Pharmacology and Experimental Therapeutics, Thomas Jefferson University, Philadelphia, PA 19107, USA. ${ }^{5}$ Current Address: Department of Neuroradiology, Technical University of Munich, Munich, Germany. ${ }^{6}$ Current Address: Leonard Davis School of Gerontology, University of Southern California, Los Angeles, CA 90089, USA

Received: 10 September 2019 Accepted: 13 February 2020 Published online: 24 February 2020

\section{References}

1. Ross CA, Poirier MA. Protein aggregation and neurodegenerative disease. Nat Med. 2004;10(Suppl):S10-7.

2. David DC, Ollikainen N, Trinidad JC, Cary MP, Burlingame AL, Kenyon C. Widespread protein aggregation as an inherent part of aging in C. elegans. PLoS Biol. 2010;8(8):e1000450.

3. Carrell RW, Lomas DA. Conformational disease. Lancet. 1997;350(9071):134-8.

4. Bucciantini M, Giannoni E, Chiti F, Baroni F, Formigli L, Zurdo J, Taddei N, Ramponi G, Dobson CM, Stefani M. Inherent toxicity of aggregates implies a common mechanism for protein misfolding diseases. Nature. 2002; 416(6880):507-11.

5. Zoghbi HY, Orr HT. Glutamine repeats and neurodegeneration. Annu Rev Neurosci. 2000;23:217-47.

6. Chen S, Ferrone FA, Wetzel R. Huntington's disease age-of-onset linked to polyglutamine aggregation nucleation. Proc Natl Acad Sci U S A. 2002; 99(18):11884-9.

7. Shao J, Diamond Ml: Polyglutamine diseases: emerging concepts in pathogenesis and therapy. Hum Mol Genet 2007, 16 Spec No. 2:R115-R123.

8. Brichta L, Greengard P. Molecular determinants of selective dopaminergic vulnerability in Parkinson's disease: an update. Front Neuroanat. 2014;8:152.

9. Fu H, Hardy J, Duff KE. Selective vulnerability in neurodegenerative diseases. Nat Neurosci. 2018;21(10):1350-8.

10. Wang YA, Kammenga JE, Harvey SC. Genetic variation in neurodegenerative diseases and its accessibility in the model organism Caenorhabditis elegans. Human Genomics. 2017;11(1):12.

11. Fiqueroa KP, Coon H, Santos N, Velazquez L, Mederos LA, Pulst SM. Genetic analysis of age at onset variation in spinocerebellar ataxia type 2. Neurol Genet. 2017;3(3):e155.

12. Wexler NS, Lorimer J, Porter J, Gomez F, Moskowitz C, Shackell E, Marder K, Penchaszadeh G, Roberts SA, Gayan J, et al. Venezuelan kindreds reveal that genetic and environmental factors modulate Huntington's disease age of onset. Proc Natl Acad Sci U S A. 2004;101(10):3498-503.

13. Gusella JF, MacDonald ME. Huntington's disease: the case for genetic modifiers. Genome Med. 2009;1 (8):80

14. Hamilton BA, Yu BD. Modifier genes and the plasticity of genetic networks in mice. PLoS Genet. 2012:8(4):e1002644.

15. Li JL, Hayden MR, Almqvist EW, Brinkman RR, Durr A, Dode C, Morrison PJ, Suchowersky O, Ross CA, Margolis RL, et al. A genome scan for modifiers of age at onset in Huntington disease: the HD MAPS study. Am J Hum Genet. 2003;73(3):682-7.

16. Li JL, Hayden MR, Warby SC, Durr A, Morrison PJ, Nance M, Ross CA, Margolis RL, Rosenblatt A, Squitieri F, et al. Genome-wide significance for a modifier of age at neurological onset in Huntington's disease at 6q23-24: the HD MAPS study. BMC Med Genet. 2006;7:71.

17. Gayan J, Brocklebank D, Andresen JM, Alkorta-Aranburu G, Group US-VCR, Zameel Cader M, Roberts SA, Cherny SS, Wexler NS, Cardon LR, et al. Genomewide linkage scan reveals novel loci modifying age of onset of Huntington's disease in the Venezuelan HD kindreds. Genet Epidemiol. 2008;32(5):445-53.

18. Long JD, Lee JM, Aylward EH, Gillis T, Mysore JS, Abu Elneel K, Chao MJ, Paulsen JS, MacDonald ME, Gusella JF. Genetic modification of Huntington disease acts early in the prediagnosis phase. Am J Hum Genet. 2018;103(3): $349-57$.

19. Genetic Modifiers of Huntington's Disease C. Identification of genetic factors that modify clinical onset of Huntington's disease. Cell. 2015;162(3):516-26.

20. Lee JM, Chao MJ, Harold D, Abu Elneel K, Gillis T, Holmans P, Jones L, Orth M, Myers RH, Kwak S, et al. A modifier of Huntington's disease onset at the MLH1 locus. Hum Mol Genet. 2017;26(19):3859-67.

21. Chao MJ, Kim KH, Shin JW, Lucente D, Wheeler VC, Li H, Roach JC, Hood L, Wexler NS, Jardim LB, et al. Population-specific genetic modification of Huntington's disease in Venezuela. PLoS Genet. 2018;14(5):e1007274.

22. Haider NB, Ikeda A, Naggert JK, Nishina PM. Genetic modifiers of vision and hearing. Hum Mol Genet. 2002;11(10):1195-206.

23. Marsh JL, Lukacsovich T, Thompson LM. Animal models of polyglutamine diseases and therapeutic approaches. J Biol Chem. 2009;284(12):7431-5.

24. Jackson GR, Salecker I, Dong X, Yao X, Arnheim N, Faber PW, MacDonald ME, Zipursky SL. Polyglutamine-expanded human huntingtin transgenes induce degeneration of Drosophila photoreceptor neurons. Neuron. 1998; 21(3):633-42.

25. Warrick JM, Paulson HL, Gray-Board GL, Bui QT, Fischbeck KH, Pittman RN, Bonini NM. Expanded polyglutamine protein forms nuclear inclusions and causes neural degeneration in Drosophila. Cell. 1998;93(6):939-49.

26. Faber PW, Alter JR, MacDonald ME, Hart AC. Polyglutamine-mediated dysfunction and apoptotic death of a Caenorhabditis elegans sensory neuron. Proc Natl Acad Sci U S A. 1999;96(1):179-84.

27. Marsh JL, Walker H, Theisen H, Zhu YZ, Fielder T, Purcell J, Thompson LM. Expanded polyglutamine peptides alone are intrinsically cytotoxic and cause neurodegeneration in Drosophila. Hum Mol Genet. 2000;9(1):13-25.

28. Kazemi-Esfarjani P. Benzer S. Genetic suppression of polyglutamine toxicity in Drosophila. Science. 2000;287(5459):1837-40. 
29. Parker JA, Connolly JB, Wellington C, Hayden M, Dausset J, Neri C. Expanded polyglutamines in Caenorhabditis elegans cause axonal abnormalities and severe dysfunction of PLM mechanosensory neurons without cell death. Proc Natl Acad Sci U S A. 2001;98(23):13318-23.

30. Morley JF, Brignull HR, Weyers JJ, Morimoto RI. The threshold for polyglutamine-expansion protein aggregation and cellular toxicity is dynamic and influenced by aging in Caenorhabditis elegans. Proc Natl Acad Sci U S A. 2002;99(16):10417-22.

31. Satyal SH, Schmidt E, Kitagawa K, Sondheimer N, Lindquist S, Kramer JM, Morimoto RI. Polyglutamine aggregates alter protein folding homeostasis in Caenorhabditis elegans. Proc Natl Acad Sci U S A. 2000;97(11):5750-5.

32. Christie NT, Lee AL, Fay HG, Gray AA, Kikis EA. Novel polyglutamine model uncouples proteotoxicity from aging. PLoS One. 2014;9(5):e96835.

33. Fardghassemi Y, Tauffenberger A, Gosselin S, Parker JA. Rescue of ATXN3 neuronal toxicity in Caenorhabditiselegans by chemical modification of endoplasmic reticulum stress. Dis Model Mech. 2017;10(12):1465-80.

34. Teixeira-Castro A, Ailion M, Jalles A, Brignull HR, Vilaca JL, Dias N, Rodrigues P, Oliveira JF, Neves-Carvalho A, Morimoto Rl, et al. Neuron-specific proteotoxicity of mutant ataxin-3 in C. elegans: rescue by the DAF-16 and HSF-1 pathways. Hum Mol Genet. 2011;20(15):2996-3009.

35. Kikis EA, Gidalevitz T, Morimoto RI. Protein homeostasis in models of aging and age-related conformational disease. Adv Exp Med Biol. 2010;694:138-59.

36. Hsu AL, Murphy $C T$, Kenyon C. Regulation of aging and age-related disease by DAF-16 and heat-shock factor. Science. 2003;300(5622):1142-5.

37. Gidalevitz T, Ben-Zvi A, Ho KH, Brignull HR, Morimoto RI. Progressive disruption of cellular protein folding in models of polyglutamine diseases. Science. 2006:311(5766):1471-4.

38. Gidalevitz T, Wang N, Deravaj T, Alexander-Floyd J, Morimoto RI. Natural genetic variation determines susceptibility to aggregation or toxicity in a $C$ elegans model for polyglutamine disease. BMC Biol. 2013;11:100.

39. Gidalevitz T, Krupinski T, Garcia S, Morimoto RI. Destabilizing protein polymorphisms in the genetic background direct phenotypic expression of mutant SOD1 toxicity. PLoS Genet. 2009;5(3):e1000399.

40. Wang YA, Snoek BL, Sterken MG, JAG R, Stastna JJ, Kammenga JE, Harvey SC. Genetic background modifies phenotypic and transcriptional responses in a C. elegans model of alpha-synuclein toxicity. BMC Genomics. 2019;20(1): 232.

41. Cook DE, Zdraljevic S, Roberts JP, Andersen EC. CeNDR, the Caenorhabditis elegans natural diversity resource. Nucleic Acids Res. 2017;45(D1):D650-7.

42. Hodgkin J, Doniach T. Natural variation and copulatory plug formation in Caenorhabditis elegans. Genetics. 1997;146(1):149-64.

43. de Bono $\mathrm{M}$, Bargmann $\mathrm{Cl}$. Natural variation in a neuropeptide $\mathrm{Y}$ receptor homolog modifies social behavior and food response in C. elegans. Cell. 1998:94(5):679-89.

44. Harvey SC, Shorto A, Viney ME. Quantitative genetic analysis of life-history traits of Caenorhabditis elegans in stressful environments. BMC Evol Biol. 2008;8:15.

45. Koch R, van Luenen HG, van der Horst M, Thijssen KL, Plasterk RH. Single nucleotide polymorphisms in wild isolates of Caenorhabditis elegans. Genome Res. 2000;10(11):1690-6.

46. Rose AM, Baillie DL, Curran J. Meiotic pairing behavior of two free duplications of linkage group I in Caenorhabditis elegans. Mol Gen Genet. 1984:195(1-2):52-6.

47. Minevich G, Park DS, Blankenberg D, Poole RJ, Hobert O. CloudMap: a cloud-based pipeline for analysis of mutant genome sequences. Genetics. 2012;192(4):1249-69.

48. Thompson OA, Snoek LB, Nijveen H, Sterken MG, Volkers RJ, Brenchley R, Van't Hof A, Bevers RP, Cossins AR, Yanai I, et al. Remarkably divergent regions punctuate the genome assembly of the Caenorhabditis elegans Hawaiian strain CB4856. Genetics. 2015;200(3):975-89.

49. Sarin S, Bertrand V, Bigelow H, Boyanov A, Doitsidou M, Poole RJ, Narula S, Hobert $\mathrm{O}$. Analysis of multiple ethyl methanesulfonate-mutagenized Caenorhabditis elegans strains by whole-genome sequencing. Genetics. 2010;185(2):417-30.

50. Seidel HS, Rockman MV, Kruglyak L. Widespread genetic incompatibility in C. elegans maintained by balancing selection. Science. 2008;319(5863):58994.

51. Sassi HE, Renihan S, Spence AM, Cooperstock RL. Gene CATCHR--gene cloning and tagging for Caenorhabditis elegans using yeast homologous recombination: a novel approach for the analysis of gene expression. Nucleic Acids Res. 2005;33(18):e163.
52. Bejsovec A, Anderson P. Myosin heavy-chain mutations that disrupt Caenorhabditis elegans thick filament assembly. Genes Dev. 1988;2(10):1307-17.

53. Barral JM, Hutagalung AH, Brinker A, Hartl FU, Epstein HF. Role of the myosin assembly protein UNC-45 as a molecular chaperone for myosin. Science. 2002;295(5555):669-71.

54. Landsverk ML, Li S, Hutagalung AH, Najafov A, Hoppe T, Barral JM, Epstein HF. The UNC-45 chaperone mediates sarcomere assembly through myosin degradation in Caenorhabditis elegans. J Cell Biol. 2007;177(2):205-10.

55. Kang C, You YJ, Avery L. Dual roles of autophagy in the survival of Caenorhabditis elegans during starvation. Genes Dev. 2007;21(17):2161-71.

56. Chapin HC, Okada M, Merz AJ, Miller DL. Tissue-specific autophagy responses to aging and stress in C. elegans. Aging. 2015;7(6):419-34.

57. Chang JT, Kumsta C, Hellman AB, Adams LM, Hansen M. Spatiotemporal regulation of autophagy during Caenorhabditis elegans aging. eLife. 2017;6: e18459.

58. Vilchez D, Morantte I, Liu Z, Douglas PM, Merkwirth C, Rodrigues AP, Manning G, Dillin A. RPN-6 determines C. elegans longevity under proteotoxic stress conditions. Nature. 2012;489(7415):263-8.

59. Lin K, Dorman JB, Rodan A, Kenyon C. daf-16: an HNF-3/forkhead family member that can function to double the life-span of Caenorhabditis elegans. Science. 1997;278(5341):1319-22.

60. Henderson ST, Johnson TE. daf-16 integrates developmental and environmental inputs to mediate aging in the nematode Caenorhabditis elegans. Curr Biol. 2001;11(24):1975-80.

61. Hajdu-Cronin YM, Chen WJ, Sternberg PW. The L-type cyclin CYL-1 and the heat-shock-factor HSF-1 are required for heat-shock-induced protein expression in Caenorhabditis elegans. Genetics. 2004;168(4):1937-49.

62. Ben-Zvi A, Miller EA, Morimoto RI. Collapse of proteostasis represents an early molecular event in Caenorhabditis elegans aging. Proc Natl Acad Sci U S A. 2009;106(35):14914-9.

63. Scherzinger E, Lurz R, Turmaine M, Mangiarini L, Hollenbach B, Hasenbank R, Bates GP, Davies SW, Lehrach H, Wanker EE. Huntingtin-encoded polyglutamine expansions form amyloid-like protein aggregates in vitro and in vivo. Cell. 1997;90(3):549-58.

64. Falsone SF, Meyer NH, Schrank E, Leitinger G, Pham CL, Fodero-Tavoletti MT, Holmberg M, Dulle M, Scicluna B, Gesslbauer B, et al. SERF protein is a direct modifier of amyloid fiber assembly. Cell Rep. 2012;2(2):358-71.

65. van Ham TJ, Holmberg MA, van der Goot AT, Teuling E, Garcia-Arencibia M, Kim HE, Du D, Thijssen KL, Wiersma M, Burggraaff R, et al. Identification of MOAG-4/SERF as a regulator of age-related proteotoxicity. Cell. 2010;142(4): $601-12$

66. Roth DM, Hutt DM, Tong J, Bouchecareilh M, Wang N, Seeley T, Dekkers JF, Beekman JM, Garza D, Drew L, et al. Modulation of the maladaptive stress response to manage diseases of protein folding. PLoS Biol. 2014;12(11): e1001998.

67. Cingolani P, Platts A, Wang le L, Coon M, Nguyen T, Wang L, Land SJ, Lu X, Ruden DM. A program for annotating and predicting the effects of single nucleotide polymorphisms, SnpEff: SNPs in the genome of Drosophila melanogaster strain w1118; iso-2; iso-3. Fly. 2012;6(2):80-92.

68. Zhang H, Wu F, Wang X, Du H, Wang X, Zhang H. The two C. elegans ATG16 homologs have partially redundant functions in the basal autophagy pathway. Autophagy. 2013;9(12):1965-74.

69. Stavoe AK, Hill SE, Hall DH, Colon-Ramos DA. KIF1A/UNC-104 transports ATG-9 to regulate neurodevelopment and autophagy at synapses. Dev Cell. 2016;38(2):171-85.

70. Mizushima N, Noda T, Yoshimori T, Tanaka Y, Ishii T, George MD, Klionsky DJ, Ohsumi M, Ohsumi Y. A protein conjugation system essential for autophagy. Nature. 1998;395(6700):395-8.

71. Mizushima N, Noda T, Ohsumi Y. Apg16p is required for the function of the Apg12p-Apg5p conjugate in the yeast autophagy pathway. EMBO J. 1999; 18(14):3888-96.

72. Melendez A, Levine B: Autophagy in C. elegans. WormBook : the online review of $C$ elegans biology 2009:1-26.

73. Yousefi S, Perozzo R, Schmid I, Ziemiecki A, Schaffner T, Scapozza L, Brunner T, Simon HU. Calpain-mediated cleavage of Atg5 switches autophagy to apoptosis. Nat Cell Biol. 2006;8(10):1124-32.

74. Zhang H, Chang JT, Guo B, Hansen M, Jia K, Kovacs AL, Kumsta C, Lapierre $L R$, Legouis $R$, Lin $L$, et al. Guidelines for monitoring autophagy in Caenorhabditis elegans. Autophagy. 2015;11(1):9-27.

75. Maiuri MC, Le Toumelin G, Criollo A, Rain JC, Gautier F, Juin P, Tasdemir E, Pierron G, Troulinaki K, Tavernarakis N, et al. Functional and physical 
interaction between $\mathrm{BCl}-\mathrm{X}(\mathrm{L})$ and a BH3-like domain in Beclin-1. EMBO J. 2007:26(10):2527-39.

76. Mohri-Shiomi A, Garsin DA. Insulin signaling and the heat shock response modulate protein homeostasis in the Caenorhabditis elegans intestine during infection. J Biol Chem. 2008;283(1):194-201.

77. Prahlad V, Morimoto RI. Neuronal circuitry regulates the response of Caenorhabditis elegans to misfolded proteins. Proc Natl Acad Sci U S A. 2011;108(34):14204-9.

78. Wang X, Wang X, Li L, Wang D. Lifespan extension in Caenorhabditis elegans by DMSO is dependent on sir-2.1 and daf-16. Biochem Biophys Res Commun. 2010:400(4):613-8.

79. Frankowski H, Alavez S, Spilman P, Mark KA, Nelson JD, Mollahan P, Rao RV, Chen SF, Lithgow GJ, Ellerby HM. Dimethyl sulfoxide and dimethyl formamide increase lifespan of C. elegans in liquid. Mech Ageing Dev. 2013; 134(3-4):69-78.

80. Ravikumar B, Duden R, Rubinsztein DC. Aggregate-prone proteins with polyglutamine and polyalanine expansions are degraded by autophagy. Hum Mol Genet. 2002;11(9):1107-17.

81. Jia K, Hart AC, Levine B. Autophagy genes protect against disease caused by polyglutamine expansion proteins in Caenorhabditis elegans. Autophagy. 2007:3(1):21-5.

82. Saxton RA, Sabatini DM. mTOR signaling in growth, metabolism, and disease. Cell. 2017;168(6):960-76.

83. Hansen M, Chandra A, Mitic LL, Onken B, Driscoll M, Kenyon C. A role for autophagy in the extension of lifespan by dietary restriction in C. elegans. PLoS Genet. 2008;4(2):e24.

84. Long X, Spycher C, Han ZS, Rose AM, Muller F, Avruch J. TOR deficiency in C. elegans causes developmental arrest and intestinal atrophy by inhibition of mRNA translation. Curr Biol. 2002;12(17):1448-61.

85. Kim DH, Sarbassov DD, Ali SM, Latek RR, Guntur KV, Erdjument-Bromage H, Tempst P, Sabatini DM. GbetaL, a positive regulator of the rapamycinsensitive pathway required for the nutrient-sensitive interaction between raptor and mTOR. Mol Cell. 2003;11(4):895-904.

86. Jones KT, Greer ER, Pearce D, Ashrafi K. Rictor/TORC2 regulates Caenorhabditis elegans fat storage, body size, and development through sgk-1. PLoS Biol. 2009;7(3):e60.

87. Melendez A, Talloczy Z, Seaman M, Eskelinen EL, Hall DH, Levine B. Autophagy genes are essential for dauer development and life-span extension in C. elegans. Science. 2003;301(5638):1387-91.

88. Cohen E, Bieschke J, Perciavalle RM, Kelly JW, Dillin A. Opposing activities protect against age-onset proteotoxicity. Science. 2006;313(5793):1604-10.

89. Mizushima N, Yamamoto A, Matsui M, Yoshimori T, Ohsumi Y. In vivo analysis of autophagy in response to nutrient starvation using transgenic mice expressing a fluorescent autophagosome marker. Mol Biol Cell. 2004; 15(3):1101-11

90. Brignull HR, Moore FE, Tang SJ, Morimoto RI. Polyglutamine proteins at the pathogenic threshold display neuron-specific aggregation in a pan-neuronal Caenorhabditis elegans model. J Neurosci. 2006;26(29): 7597-606.

91. Maday S, Holzbaur EL. Compartment-specific regulation of autophagy in primary neurons. J Neurosci. 2016;36(22):5933-45.

92. Nikoletopoulou V, Markaki M, Palikaras K, Tavernarakis N. Crosstalk between apoptosis, necrosis and autophagy. Biochim Biophys Acta. 2013;1833(12): 3448-59.

93. Ding WX, Ni HM, Gao W, Hou YF, Melan MA, Chen X, Stolz DB, Shao ZM, Yin $X M$. Differential effects of endoplasmic reticulum stress-induced autophagy on cell survival. J Biol Chem. 2007;282(7):4702-10.

94. Nixon RA. The role of autophagy in neurodegenerative disease. Nat Med. 2013;19(8):983-97.

95. Ciechanover A, Kwon YT. Degradation of misfolded proteins in neurodegenerative diseases: therapeutic targets and strategies. Exp Mol Med. 2015:47:e147.

96. Menzies FM, Fleming A, Caricasole A, Bento CF, Andrews SP, Ashkenazi A, Fullgrabe J, Jackson A, Jimenez Sanchez M, Karabiyik C, et al. Autophagy and neurodegeneration: pathogenic mechanisms and therapeutic opportunities. Neuron. 2017:93(5):1015-34.

97. Zielonka D, Piotrowska I, Marcinkowski JT, Mielcarek M. Skeletal muscle pathology in Huntington's disease. Front Physiol. 2014;5:380.

98. Huang S, Yang S, Guo J, Yan S, Gaertig MA, Li S, Li XJ. Large polyglutamine repeats cause muscle degeneration in SCA17 mice. Cell Rep. 2015;13(1): 196-208.
99. Luthi-Carter R, Hanson SA, Strand AD, Bergstrom DA, Chun W, Peters NL, Woods AM, Chan EY, Kooperberg C, Krainc D, et al. Dysregulation of gene expression in the R6/2 model of polyglutamine disease: parallel changes in muscle and brain. Hum Mol Genet. 2002;11(17):1911-26.

100. Moffitt H, McPhail GD, Woodman B, Hobbs C, Bates GP. Formation of polyglutamine inclusions in a wide range of non-CNS tissues in the HdhQ150 knock-in mouse model of Huntington's disease. PLoS One. 2009; 4(11):e8025.

101. She P, Zhang Z, Marchionini D, Diaz WC, Jetton TJ, Kimball SR, Vary TC, Lang $\mathrm{CH}$, Lynch CJ. Molecular characterization of skeletal muscle atrophy in the R6/2 mouse model of Huntington's disease. Am J Physiol Endocrinol Metab. 2011;301(1):E49-61.

102. Zuryn S, Le Gras S, Jamet K, Jarriault S. A strategy for direct mapping and identification of mutations by whole-genome sequencing. Genetics. 2010; 186(1):427-30.

103. Rutherford SL, Lindquist S. Hsp90 as a capacitor for morphological evolution. Nature. 1998:396(6709):336-42.

104. Gibson G. Decanalization and the origin of complex disease. Nat Rev Genet. 2009:10(2):134-40.

105. Paaby AB, Rockman MV. Cryptic genetic variation: evolution's hidden substrate. Nat Rev Genet. 2014;15(4):247-58.

106. Luthi-Carter R, Strand AD, Hanson SA, Kooperberg C, Schilling G, La Spada $A R$, Merry DE, Young AB, Ross CA, Borchelt DR, et al. Polyglutamine and transcription: gene expression changes shared by DRPLA and Huntington's disease mouse models reveal context-independent effects. Hum Mol Genet. 2002:11(17):1927-37.

107. Goold R, Flower M, Moss DH, Medway C, Wood-Kaczmar A, Andre R, Farshim P, Bates GP, Holmans P, Jones L, et al. FAN1 modifies Huntington's disease progression by stabilizing the expanded HTT CAG repeat. Hum Mol Genet. 2019;28(4):650-61.

108. Walker DW, McColl G, Jenkins NL, Harris J, Lithgow GJ. Evolution of lifespan in C. elegans. Nature. 2000;405(6784):296-7.

109. Jenkins NL, McColl G, Lithgow GJ. Fitness cost of extended lifespan in Caenorhabditis elegans. Proc Biol Sci. 2004;271(1556):2523-6.

110. Chen J, Senturk D, Wang JL, Muller HG, Carey JR, Caswell H, Caswell-Chen EP. A demographic analysis of the fitness cost of extended longevity in Caenorhabditis elegans. J Gerontol A Biol Sci Med Sci. 2007;62(2):126-35.

111. Altman BJ, Rathmell JC. Metabolic stress in autophagy and cell death pathways. Cold Spring Harb Perspect Biol. 2012;4(9):a008763.

112. Lu K, Psakhye I, Jentsch S. Autophagic clearance of polyQ proteins mediated by ubiquitin-Atg8 adaptors of the conserved CUET protein family. Cell. 2014;158(3):549-63.

113. Martinez-Vicente M, Talloczy Z, Wong E, Tang G, Koga H, Kaushik S, de Vries $R$, Arias $E$, Harris $S$, Sulzer $D$, et al. Cargo recognition failure is responsible for inefficient autophagy in Huntington's disease. Nat Neurosci. 2010;13(5):567-76.

114. Schiaffino S, Dyar KA, Ciciliot S, Blaauw B, Sandri M. Mechanisms regulating skeletal muscle growth and atrophy. FEBS J. 2013;280(17):4294-314.

115. Mammucari C, Milan G, Romanello V, Masiero E, Rudolf R, Del Piccolo P, Burden SJ, Di Lisi R, Sandri C, Zhao J, et al. FoxO3 controls autophagy in skeletal muscle in vivo. Cell Metab. 2007:6(6):458-71.

116. Zhao J, Brault JJ, Schild A, Cao P, Sandri M, Schiaffino S, Lecker SH, Goldberg AL. FoxO3 coordinately activates protein degradation by the autophagic/ lysosomal and proteasomal pathways in atrophying muscle cells. Cell Metab. 2007;6(6):472-83.

117. Masiero E, Agatea L, Mammucari C, Blaauw B, Loro E, Komatsu M, Metzger D, Reggiani C, Schiaffino S, Sandri M. Autophagy is required to maintain muscle mass. Cell Metab. 2009;10(6):507-15.

118. Heiman-Patterson TD, Sher RB, Blankenhorn EA, Alexander G, Deitch JS, Kunst CB, Maragakis N, Cox G. Effect of genetic background on phenotype variability in transgenic mouse models of amyotrophic lateral sclerosis: a window of opportunity in the search for genetic modifiers. Amyotroph Lateral Scler. 2011;12(2):79-86.

119. Peters TW, Nelson CS, Gerencser AA, Dumas KJ, Tavshanjian B, Chang KC, Lithgow GJ, Hughes RE. Natural genetic variation in yeast reveals that NEDD4 is a conserved modifier of mutant polyglutamine aggregation. G3. 2018;8(11):3421-31.

120. Lloret A, Dragileva E, Teed A, Espinola J, Fossale E, Gillis T, Lopez E, Myers RH, MacDonald ME, Wheeler VC. Genetic background modifies nuclear mutant huntingtin accumulation and HD CAG repeat instability in Huntington's disease knock-in mice. Hum Mol Genet. 2006;15(12):2015-24. 
121. Van Raamsdonk JM, Metzler M, Slow E, Pearson J, Schwab C, Carroll J, Graham RK, Leavitt BR, Hayden MR. Phenotypic abnormalities in the YAC128 mouse model of Huntington disease are penetrant on multiple genetic backgrounds and modulated by strain. Neurobiol Dis. 2007;26(1):189-200.

122. Chow CY, Wolfner MF, Clark AG. Using natural variation in Drosophila to discover previously unknown endoplasmic reticulum stress genes. Proc Natl Acad Sci U S A. 2013;110(22):9013-8.

123. Mackay TF, Richards S, Stone EA, Barbadilla A, Ayroles JF, Zhu D, Casillas S, Han Y, Magwire MM, Cridland JM, et al. The Drosophila melanogaster genetic reference panel. Nature. 2012;482(7384):173-8.

124. He BZ, Ludwig MZ, Dickerson DA, Barse L, Arun B, Vilhjalmsson BJ, Jiang P, Park SY, Tamarina NA, Selleck SB, et al. Effect of genetic variation in a Drosophila model of diabetes-associated misfolded human proinsulin. Genetics. 2014;196(2):557-67.

125. Brenner S. The genetics of Caenorhabditis elegans. Genetics. 1974:77(1):71-94.

126. Afgan $E$, Baker D, van den Beek $M$, Blankenberg D, Bouvier D, Cech $M$, Chilton J, Clements D, Coraor N, Eberhard C, et al. The galaxy platform for accessible, reproducible and collaborative biomedical analyses: 2016 update. Nucleic Acids Res. 2016;44(W1):W3-W10.

127. Schneider CA, Rasband WS, Eliceiri KW. NIH image to ImageJ: 25 years of image analysis. Nat Methods. 2012;9(7):671-5.

128. Livak KJ, Schmittgen TD. Analysis of relative gene expression data using real-time quantitative PCR and the 2(-Delta Delta C(T)) Method. Methods. 2001;25(4):402-8

129. Lascarez-Lagunas LI, Silva-Garcia CG, Dinkova TD, Navarro RE. LIN-35/Rb causes starvation-induced germ cell apoptosis via CED-9/BCl2 downregulation in Caenorhabditis elegans. Mol Cell Biol. 2014;34(13):2499-516.

130. Alexander-Floyd J, Gidalevitz T: SNPs in the C. elegans drxIR1;Q40 strain. NCBI Sequence Read Archive, Accession: PRJNA598355( https://www.ncbi. nlm.nih.gov/sra/PRJNA598355 ) 2020.

131. Lee RYN, Howe KL, Harris TW, Arnaboldi V, Cain S, Chan J, Chen WJ, Davis P, Gao S, Grove C, et al. WormBase 2017: molting into a new stage. Nucleic Acids Res. 2018;46(D1):D869-74.

132. Shaye DD, Greenwald I. OrthoList: a compendium of C. elegans genes with human orthologs. PloS one. 2011;6(5):e20085.

\section{Publisher's Note}

Springer Nature remains neutral with regard to jurisdictional claims in published maps and institutional affiliations.

Ready to submit your research? Choose BMC and benefit from:

- fast, convenient online submission

- thorough peer review by experienced researchers in your field

- rapid publication on acceptance

- support for research data, including large and complex data types

- gold Open Access which fosters wider collaboration and increased citations

- maximum visibility for your research: over $100 \mathrm{M}$ website views per year

At $\mathrm{BMC}$, research is always in progress.

Learn more biomedcentral.com/submissions 